\title{
A family of residual-based stabilized finite element methods for Stokes flows
}

\author{
Eugenio Oñate $^{1 *}$, Prashanth Nadukandi ${ }^{1}$, Sergio R. Idelsohn ${ }^{2}$, Julio García ${ }^{1}$ \\ and Carlos Felippa ${ }^{3}$
}

\author{
${ }^{1}$ International Center for Numerical Methods in Engineering (CIMNE) \\ Technical University of Catalonia (UPC) \\ Campus Norte UPC, 08034 Barcelona, Spain \\ www.cimne.com/eo, onate@cimne.upc.edu \\ ${ }^{2}$ ICREA Research Professor at CIMNE \\ ${ }^{3}$ Department of Aerospace Engineering Sciences and Center for Aerospace Structures \\ University of Colorado, Boulder, CO $80309-0429$, USA \\ Visiting Professor at CIMNE
}

\begin{abstract}
SUMMARY
We present a collection of stabilized finite element (FE) methods derived via first and second order finite calculus (FIC) procedures. It is shown that several well known existing stabilized FE methods such as the penalty technique, the Galerkin Least Square (GLS) method, the Pressure Gradient Projection (PGP) method and the orthogonal sub-scales (OSS) method are recovered from the general residual-based FIC stabilized form. A new family of stabilized Pressure-Laplacian Stabilization (PLS) FE methods with consistent nonlinear forms of the stabilization parameters are derived. The distinct feature of the family of PLS methods is that they are residual-based, i.e. the stabilization terms depend on the discrete residuals of the momentum and/or the incompressibility equations. The advantages and disadvantages of the different stabilization techniques are discussed and several examples of application are presented.
\end{abstract}

KEY WORDS: Stabilized finite element method, Stokes flows, finite calculus, Pressure Laplacian Stabilization

\section{INTRODUCTION}

Many stabilization procedures for solving incompressible problems in fluid mechanics using the finite element method (FEM) have been proposed [1]-[28]. Earlier procedures were based on the so-called penalty approach. This method assumes a pseudo-compressible behaviour for the flow with a relationship between the volumetric strain rate $\varepsilon_{v}$ and the pressure $p$ expressed as $[1,2]$

$$
\varepsilon_{v}=\frac{1}{\alpha} p
$$

where $\alpha$ is a large number playing the role of an "artificial" bulk parameter for the fluid. Clearly for $\alpha \rightarrow \infty$ the full incompressibility condition $\varepsilon_{v} \rightarrow 0$ is recovered. Another family of stabilized methods added to the standard incompressibility equation (either in the strong form or in the variational expression) a Laplacian of pressure term scaled by a stabilization coefficient depending

*Correspondence to: CIMNE, Edificio C1, Campus Norte, UPC, Gran Capitán s/n, 08034 Barcelona, Spain 
on physical parameters and the time step increment. Some of these stabilization methods are described in $[1,2]$. A similar stabilization procedure adds to the variational equation a local $L_{2}$ polynomial pressure projection multiplied by the inverse of the kinematic viscosity [19]. These approaches are inconsistent since the stabilization term does not vanish for the exact solution, which can lead to errors in the pressure distribution and in the conservation of the total volume for some problems. An improved stabilization technique adds to the incompressibility condition a term that is a function of the discretized momentum equations, thus ensuring consistency. A popular procedure of this kind is the Galerkin Least Square (GLS) method [4, 8]. In the GLS procedure the stabilized variational expression for the incompressibility equation has the following form

$$
\int_{\Omega} q \varepsilon_{v} d \Omega-\int_{\Omega} \tau\left(\nabla^{T} q\right) \overline{\mathbf{r}}_{m} d \Omega+\text { boundary terms }=0
$$

where $\Omega$ is the analysis domain, $q$ are test functions, $\nabla$ is the gradient operator, $\tau$ is a stabilization parameter and $\overline{\mathbf{r}}_{m}$ is a vector containing the discrete residuals of the momentum equations written as $r_{m}=0$. The boundary terms in Eq.(2) are added to ensure the consistency of the method [8]. The GLS method is an efficient and accurate stabilization procedure for incompressible flows provided the boundary terms are properly accounted for in Eq.(2).

Another residual-based stabilization technique is the so-called pressure-gradient projection (PGP) stabilization $[15,16])$. In the PGP method, pressure gradients are projected onto a continuous field and the difference between the actual gradients and their own projections generates the stabilization terms. This is equivalent to replacing the variational expression for the incompressibility equation by the following equation
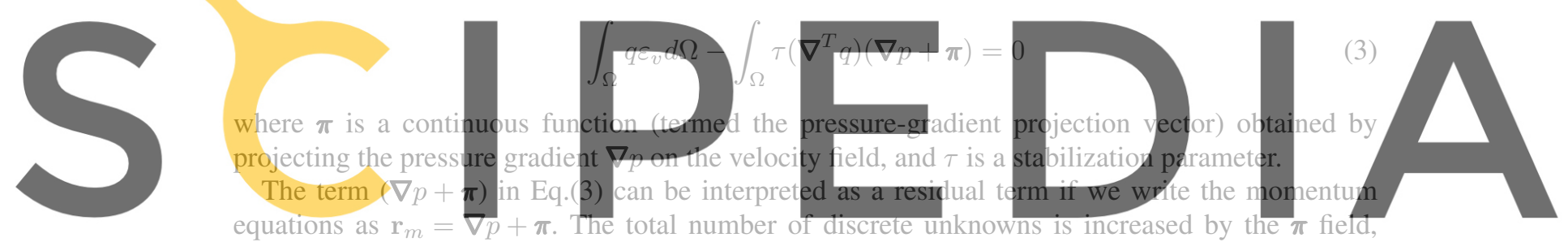

which is discretized via pressure shape functions. For completeness, the set of governing equations

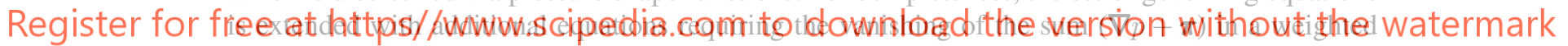
residual sense. This provides the equations for computing the $\pi$ variables.

A variant of the PGP technique is the orthogonal sub-scales (OSS) method [17, 20, 21]. The

variational expression for the incompressibility constraint is written in the OSS method as

$$
\int_{\Omega} q \varepsilon_{v} d \Omega-\int_{\Omega} \tau\left(\nabla^{T} q\right)\left(\overline{\mathbf{r}}_{m}+\boldsymbol{\pi}\right) d \Omega=0
$$

where $\overline{\mathbf{r}}_{m}$ is the discrete residual of the momentum equations and $\boldsymbol{\pi}$ are additional variables that are now interpreted as the projection of the momentum residuals into the velocity space (without boundary conditions). The term $\overline{\mathbf{r}}_{m}+\boldsymbol{\pi}$ represents an enhanced approximation to the exact momentum residuals $\mathbf{r}_{m}$. Consistency is preserved by enforcing that the sum $\overline{\mathbf{r}}_{m}+\boldsymbol{\pi}$ vanishes in a weighted residual sense. This also provides the closure equations for computing the $\boldsymbol{\pi}$ variables.

PGP and OSS stabilization methods are useful for homogeneous flows lacking free-surfaces but encounter difficulties to satisfy incompressibility for fluids with heterogeneous (and discontinous) physical properties $[39,44,45]$ and, in some cases, for free-surface flows when pressure segregation techniques are used for solving the Navier-Stokes equations. Furthermore, PGP and OSS methods increase the number of problem variables $(\mathbf{u}, p$ and $\boldsymbol{\pi})$ as well as the connectivity (bandwidth) of the stabilization matrices to be solved.

The stabilization parameter $\tau$ in the GLS, PGP and OSS methods is typically chosen as a function of the viscosity and the mesh size [1]-[28]. However, the optimal definition of the stabilization parameter is still a challenge in these methods.

In a recent work we have presented a Pressure Laplacian Stabilization (PLS) method [46], that adds two stabilization terms to the variational form of the incompressibility equation: (1) a 
pressure Laplacian, and (2) a boundary integral. Both terms are multiplied by residual dependent stabilization parameters which emerge naturally from the formulation. Consistency is preserved since the stabilization parameters vanish for the exact solution. The Laplace matrix and the boundary matrix are computed at element level. Because pressure gradient continuity is not enforced, as it happens, for instance, in standard PGP methods, the treatment of heterogeneous multi-fluid problems, such as mixing, is facilitated.

Inclusion of the boundary term in the PLS method also sidesteps the need for prescribing known boundary pressure values when a segregated solution procedure is used. This helps to ensuring the overall conservation of mass for free surface flows [43].

The aim of the paper is to show that many of the stabilized methods described in the previous lines, and some new ones, can be derived starting from the modified mass balance equation obtained via first and second order finite calculus (FIC) procedures. The FIC technique is based on writing the balance equations in mechanics in a domain of finite size and retaining higher order terms in the Taylor series expansions used for expressing the derivative field in the vicinity of a fixed point in the domain. The resulting modified balance equation contains the traditional terms of infinitesimal theory plus additional terms that depend on the dimensions of the balance domain and the derivatives of the infinitesimal balance equations [22]. Clearly, as the dimensions of the balance domain tend to zero the classical balance laws of mechanics are recovered. The interest of the additional terms in the FIC expressions is that they lead naturally the stabilized numerical schemes (such as stabilized FEM) in fluid and solid mechanics without the need of introducing additional assumptions [22, 23], [29]-[37]. The FIC approach therefore is presented here as a parent procedure for deriving a family of old and new residual-based stabilized methods for analysis of Stokes flows.

An apparent drawback of some of the residual-based stabilized methods presented in this paper is

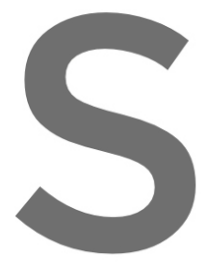
that the resulting stabil parameters) and this simple Stokes flow PLS solution is typical within a time integration scheme in transient pro linearities might appear due to the presence of
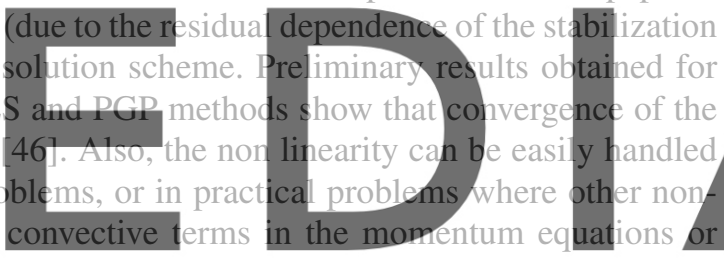

non-linear material behaviour. In the last part of the paper, the performance of the PLS method is

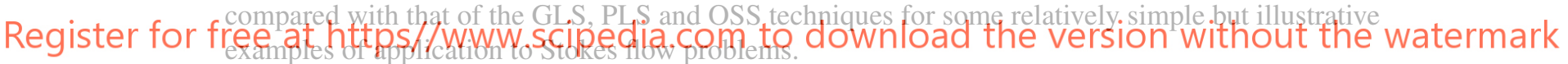

\section{GOVERNING EQUATIONS}

The equations for an incompressible Stokes flow are expressed in the usual manner as:

\section{Momentum}

$$
\rho \frac{D v_{i}}{D t}-\frac{\partial \sigma_{i j}}{\partial x_{j}}-b_{i}=0 \quad \text { on } \Omega
$$

Mass balance (incompressibility)

$$
\varepsilon_{v}:=\frac{\partial v_{i}}{\partial x_{i}}=0 \quad \text { on } \Omega \quad, \quad i=1,2,3
$$

In Eqs.(5) and (6), $\Omega$ is the analysis domain with a boundary $\Gamma, v_{i}$ is the velocity along the $i$ th coordinate direction, $\rho$ is the density, $\sigma_{i j}$ are the Cauchy stresses, $b_{i}$ are the body forces (typically $b_{i}=\rho g_{i}$ where $g_{i}$ is the component of the gravity along the $i$ th direction). In our work we assume that $\frac{D v_{i}}{D t}=\frac{\partial v_{i}}{\partial t}$, i.e. convective derivative terms are neglected, as it is usual in Stokes flows and Lagrangian descriptions of incompressible continua [32]-[34],[38, 41, 42].

The problem is completed with the boundary conditions for velocities and tractions, i.e.

$$
\begin{array}{rll}
v_{i}-v_{i}^{p} & =0 & \text { on } \Gamma_{u} \\
\sigma_{i j} n_{j}-t_{i}^{p} & =0 & \text { on } \Gamma_{t}
\end{array}
$$


where $v_{i}^{p}$ denote the prescribed velocities on the Dirichlet boundary $\Gamma_{u}$ and $t_{i}^{p}$ are the traction forces acting on the Neuman boundary $\Gamma_{t}$, with the normal vector $\mathbf{n}=\left[n_{1}, n_{2}, n_{3}\right]^{T}$ (for $3 \mathrm{D}$ problems). The total boundary is $\Gamma:=\Gamma_{u} \cup \Gamma_{t}$.

In Eqs.(5)-(7) and in the following, summation convention for repeated indices in products and derivatives is used unless otherwise specified.

Following standard practice, Cauchy stresses are split into deviatoric and pressure components as

$$
\sigma_{i j}=s_{i j}+p \delta_{i j}
$$

where $s_{i j}$ are deviatoric stresses, $p=\sigma_{i i} / 3$ is the pressure (assumed here to be positive if the mean normal stress is tensile) and $\delta_{i j}$ is the Kroneker delta.

We will also assume the constitutive equations of an isotropic, Newtonian viscous liquid for which deviatoric stresses are related to deformation rates $\varepsilon_{i j}$ by

$$
s_{i j}=2 \mu\left(\varepsilon_{i j}-\frac{1}{3} \varepsilon_{v} \delta_{i j}\right)
$$

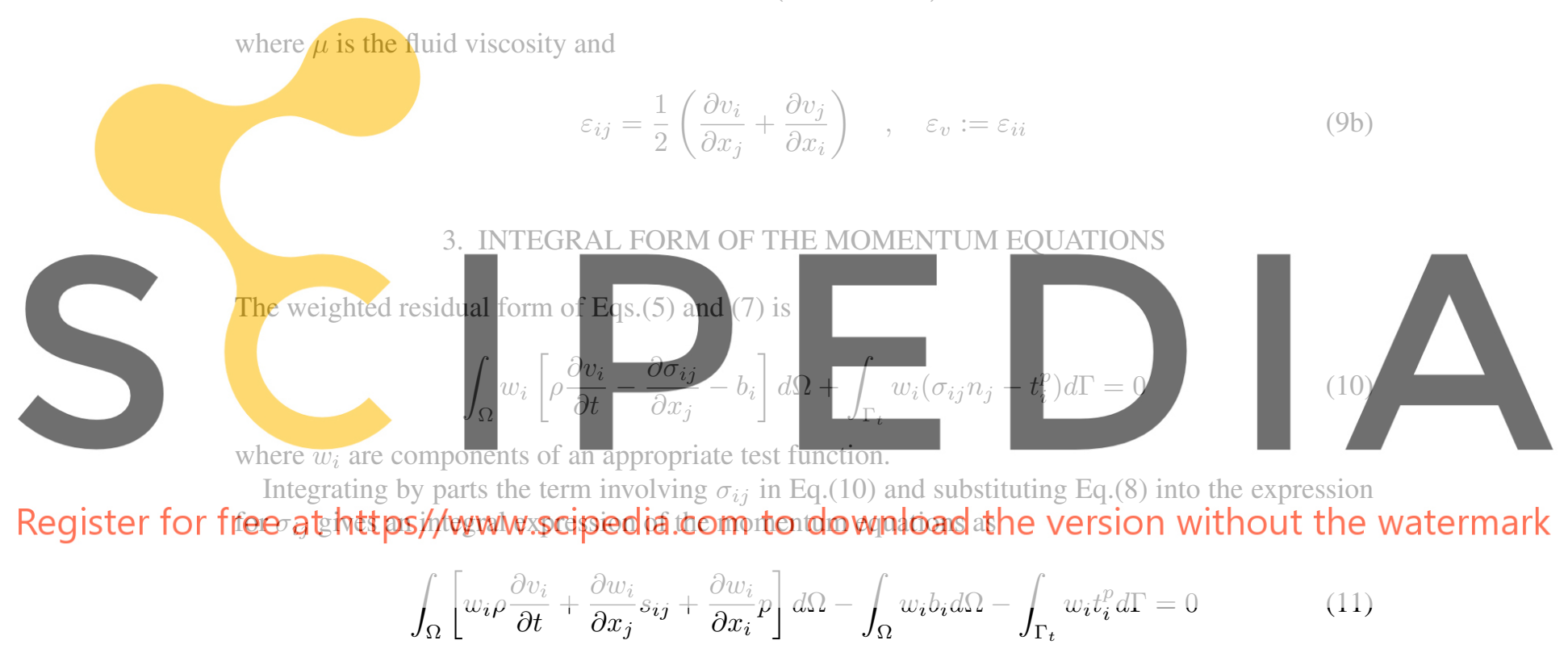

Eq.(11) is the starting point for the finite element discretization of the momentum equations.

\section{STABILIZED FORM OF THE INCOMPRESSIBILITY EQUATION USING FINITE CALCULUS}

The Finite Calculus (FIC) form of the incompressibility equation is obtained by writing the balance of mass in a finite size domain of dimension $h_{1} \times h_{2}$ where $h_{i}$ are characteristic length distances. Higher order Taylor series for expressing the velocity derivative at a reference point in terms of the velocity derivative at the corners of the balance domain is used[22, 23, 31].

We present next two stabilized forms for the mass balance equation using first and second order FIC techniques.

\subsection{First order FIC form of the incompressibility equation}

The first order FIC form for the incompressibility equation is found by writting the balance of mass in the finite domain of Figure 1a where the velocity field is assumed to vary linearly over each side. The velocity derivatives at the corner nodes are expressed in terms of the values sampled at the corners 1 or 3 via Taylor series and retaining the second derivatives of the velocities only. The 


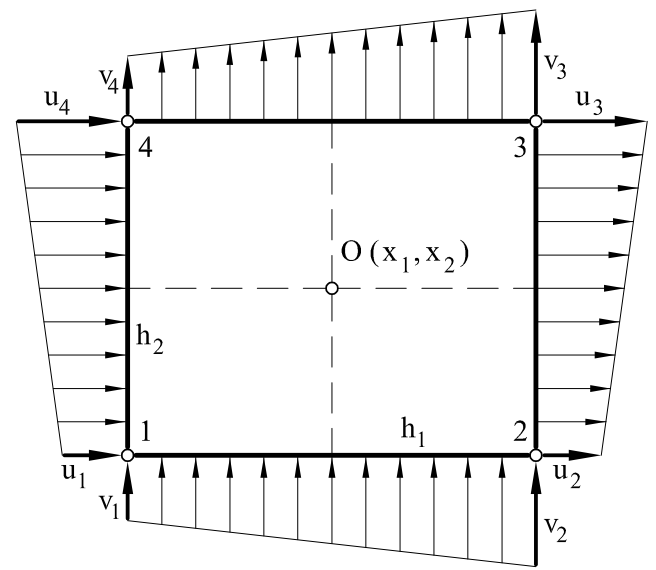

(a)

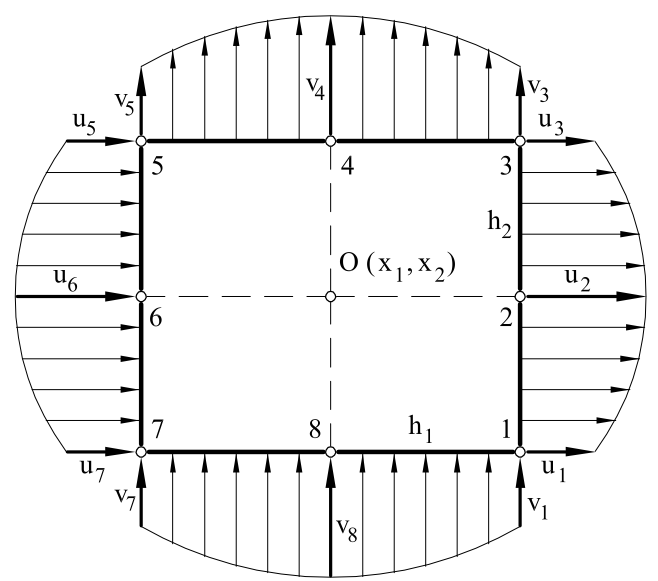

(b)

Figure 1. Rectangular mass balance domain $\left(h_{1} \times h_{2}\right)$. (a) Linear distribution of the velocity along the sides.

(b) Parabolic distribution of the velocity along the sides. For simplicity we have defined $v_{1}=u, v_{2}=v$

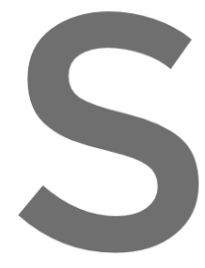

restling expression is

\section{Register for free at https//www.scipedia.com to download the version without the watermark}

\subsection{Higher order FIC form of the incompressibility equation}

A higher order FIC form for the incompressibility equation is obtained by expressing the balance of mass in the finite domain of Figure $1 \mathrm{~b}$ in terms of the value of the velocity derivatives sampled at the center of the domain. Note that the velocity field is now assumed to have a parabolic distribution along the sides.

The higher order FIC incompressibility equation includes second order derivatives of the volumetric strain rate only and it is written for $2 \mathrm{D}$ problems as

$$
\varepsilon_{v}+\frac{h_{1}^{2}}{24} \frac{\partial^{2} \varepsilon_{v}}{\partial x_{1}^{2}}+\frac{h_{2}^{2}}{24} \frac{\partial^{2} \varepsilon_{v}}{\partial x_{2}^{2}}=0
$$

Clearly for the infinitesimal case $h_{1}=h_{2}=0$ and the standard incompressibility equation $\left(\varepsilon_{v}=\right.$ 0 ) is recovered for both Eqs.(12) and (13).

Eqs.(12) and (13) can be interpreted as non-local mass balance equations incorporating the size of the domain used to enforce the mass balance condition and space derivatives of the volumetric strain rate. The FIC mass balance equations can be extended to account for temporal stabilization terms. These terms, however, are disregarded here as they have not been found to be relevant for the problems investigated so far.

The derivation of Eq.(13) is shown in [46]. 


\section{ON THE PROPORTIONALITY BETWEEN THE PRESSURE AND THE VOLUMETRIC STRAIN RATE}

Let us assume a relationship between the pressure and the volumetric strain rate typical for "compressible" and "quasi-incompressible" fluids, as

$$
\frac{1}{K} p=\varepsilon_{v}
$$

where $K$ is the bulk modulus. Clearly for a fully incompressible fluid $K=\infty$ and $\varepsilon_{v}=0$. For finite, although very large, values of $K$ the following expression is readily deduced from Eq.(14)

$$
\frac{1}{K} \nabla p=\nabla \varepsilon_{v}
$$

where $\boldsymbol{\nabla}$ is the gradient operator. For 2D problems, $\boldsymbol{\nabla}=\left\lceil\frac{\partial}{\partial x_{1}}, \frac{\partial}{\partial x_{2}}\right]^{T}$.

Eq.(15) shows that pressure and volumetric strain rate gradients are co-directional for any $K \neq 0$. We will assume that this property also holds for the full incompressible case (at least for values of $K$ comfortably representable on the computer without overflow). From Eqs.(14) and (15) we deduce

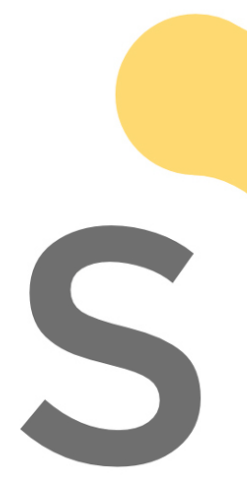

$$
\frac{\nabla \varepsilon_{v}}{\left|\nabla \varepsilon_{v}\right|}=\frac{\nabla p}{|\nabla p|} \quad, \quad \frac{1}{\varepsilon_{v}} \frac{\partial \varepsilon_{v}}{\partial x_{i}}=\frac{1}{p} \frac{\partial p}{\partial x_{i}}
$$

(16a)
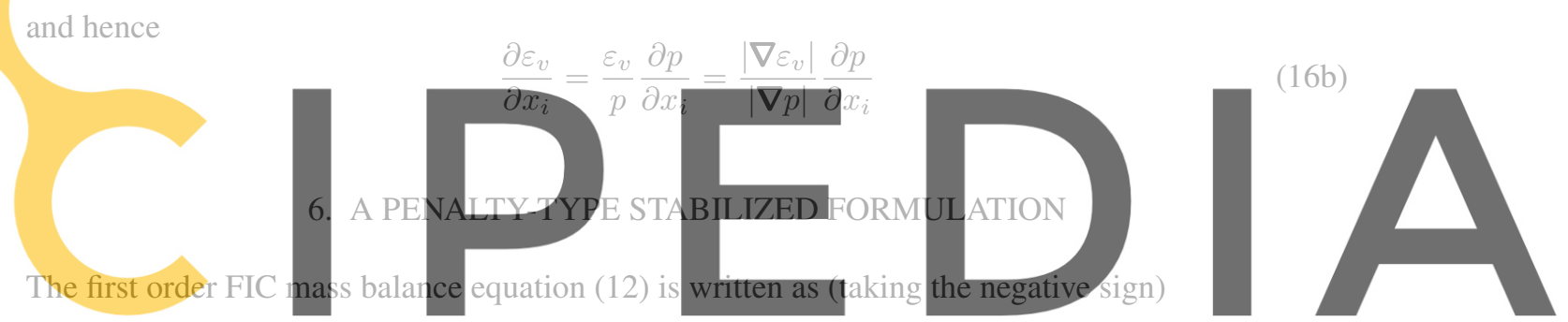

\section{Register for free at https//www.scipedia.com to elownload the version without the watermark}

Using Eq.(16b), the FIC term in the r.h.s. of Eq.(17) can be expressed as follows

$$
\varepsilon_{v}=\left(\frac{h_{j}}{2} \frac{\varepsilon_{v}}{p^{2}} \frac{\partial p}{\partial x_{j}}\right) p=\frac{1}{\alpha} p
$$

where

$$
\alpha=\frac{2 p^{2}}{h_{j} \varepsilon_{v}}\left(\frac{\partial p}{\partial x_{j}}\right)^{-1}
$$

is a pressure stabilization parameter $[1,2,41]$.

Eq.(18) is equivalent to the so-called penalty formulation (see Eq.(1)) for which the pressurevolumetric strain rate relationship is expressed as $p=\alpha \varepsilon_{v}$ where $\alpha$ a penalty parameter that plays the role of a large "artificial" bulk modulus.

Clearly, $\alpha \rightarrow \infty$ for values of $\varepsilon_{v} \rightarrow 0$. However, the full incompressibility condition $\left(\varepsilon_{v}=0\right)$ at element level is obtained on rare occasions only. Hence, the form for $\alpha$ of Eq.(19) provides a consistent (residual-based) definition for the penalty stabilization parameter. Nevertheless, upper and lower cut-off values for the values for $\alpha$ should be imposed to prevent volumetric locking when $\varepsilon_{v}$ or $\frac{\partial p}{\partial x_{i}}$ are equal to zero or the vanishing of $\alpha$ in zones where $p$ is zero $[1,2,41]$.

The weighed residual form of Eq.(18) is

$$
\int_{\Omega} q\left(\varepsilon_{v}-\frac{1}{\alpha} p\right) d \Omega=0
$$

where $q$ are adequate test functions. 


\section{GALERKIN-LEAST SQUARES (GLS) FORMULATION}

The starting point is now the higher order FIC incompressibility equation (13). The weighted residual form of this equation is

$$
\int_{\Omega} q\left(\varepsilon_{v}+\frac{h_{i}^{2}}{24} \frac{\partial^{2} \varepsilon_{v}}{\partial x_{i}^{2}}\right) d \Omega=0
$$

Integration by parts of the second term in Eq.(21) gives (for 2D problems)

$$
\int_{\Omega} q \varepsilon_{v} d \Omega-\int_{\Omega}\left(\sum_{i=1}^{2} \frac{h_{i}^{2}}{24} \frac{\partial q}{\partial x_{i}} \frac{\partial \varepsilon_{v}}{\partial x_{i}}\right) d \Omega+\int_{\Gamma} \frac{q}{24}\left(\sum_{i=1}^{2} n_{i} h_{i}^{2} \frac{\partial \varepsilon_{v}}{\partial x_{i}}\right) d \Gamma=0
$$

where $n_{i}$ are the components of the unit normal vector to the boundary $\Gamma$.

In the derivation of Eq.(22), space derivatives of the characteristic lengths $h_{1}$ and $h_{2}$ have been neglected. This is correct if we assume that the value of the characteristic lengths is fixed at each point in space. In any case, this assumption does not invalidate the derivation, as long as the discretized formulation converges to correct velocity and pressure fields satisfying the momentum and incompressibility equations in a weighted residual sense and up to the desired order of accuracy.

The term $\frac{\partial \varepsilon_{v}}{\partial x_{i}}$ in Eq.(22) is expressed as follows. The momentum equations (5) can be written using Eqs.(8) and (9a)

$$
\rho \frac{\partial v_{i}}{\partial t}-\frac{\partial}{\partial x_{i}}\left(2 \mu \varepsilon_{i j}\right)+\frac{2}{3} \mu \frac{\partial \varepsilon_{v}}{\partial x_{i}}-\frac{\partial p}{\partial x_{i}}-b_{i}=0
$$
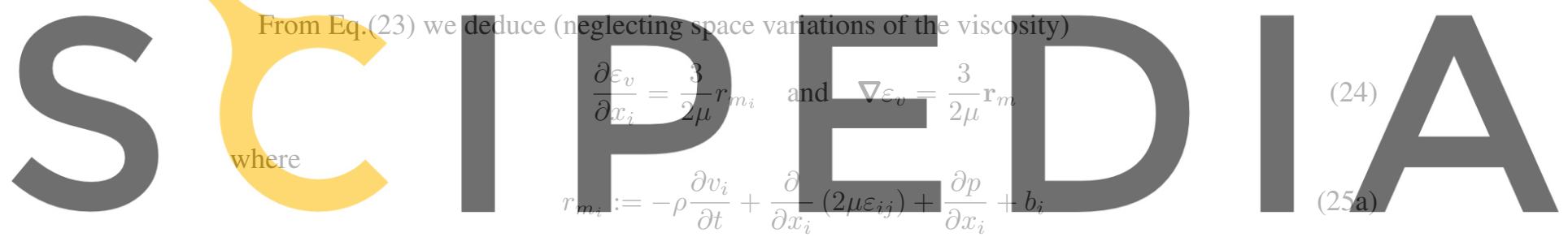

is the form of the momentum residuals used in the subsequent derivations. Note that $r_{m}=0$ for the

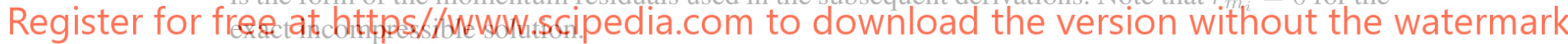

For 2D problems

$$
r_{m}=\left[r_{m_{1}}, r_{m_{2}}\right]^{T}
$$

Substituting $\frac{\partial \varepsilon_{v}}{\partial x_{i}}$ from Eq.(24) into (22) gives

$$
\int_{\Omega} q \varepsilon_{v} d \Omega-\int_{\Omega}\left(\sum_{i=1}^{2} \tau_{i} \frac{\partial q}{\partial x_{i}} r_{m_{i}}\right) d \Omega+\int_{\Gamma} q\left(\sum_{i=1}^{2} \tau_{i} n_{i} r_{m_{i}}\right) d \Gamma=0
$$

with

$$
\tau_{i}=\frac{h_{i}^{2}}{16 \mu}
$$

The form of Eq.(26) is equivalent to that obtained in the Galerkin Least Square (GLS) formulation [4] with the boundary integral modification presented in [8]. The expression for the stabilization $\tau_{i}$ of Eq.(27) is similar to that typically found in the stabilization literature for Stokes flows $[1,2,4,8]$.

Expansion of the residual term within the second integral yields the standard Laplacian of pressure plus additional terms, i.e.

$$
\begin{aligned}
& \int_{\Omega} q \varepsilon_{v} d \Omega-\int_{\Omega}\left(\sum_{i=1}^{2} \tau_{i} \frac{\partial q}{\partial x_{i}} \frac{\partial p}{\partial x_{i}}\right) d \Omega-\int_{\Omega} \sum_{i=1}^{2}\left(\tau_{i} \frac{\partial q}{\partial x_{i}}\left[-\rho \frac{\partial v_{i}}{\partial t}+\frac{\partial}{\partial x_{j}}\left(2 \mu \varepsilon_{i j}\right)+b_{i}\right]\right) d \Omega \\
& +\int_{\Gamma} q\left(\sum_{i=1}^{2} \tau_{i} n_{i}\left[-\rho \frac{\partial v_{i}}{\partial t}+\frac{\partial p}{\partial x_{j}}+\frac{\partial}{\partial x_{i}}\left(2 \mu \varepsilon_{i j}\right)+b_{i}\right]\right) d \Gamma=0
\end{aligned}
$$


Clearly for linear FE approximations the viscous terms vanish in Eq.(28).

We note that the FIC approach presented here introduces the GLS-type stabilization terms just in the incompressibility equation. This is a difference with the standard GLS method which also introduces stabilization terms in the momentum equations [4]. These terms provide symmetry of the global system of equations and are useful for analysis of Navier-Stokes flows. However, they are typically unnecessary for analysis of Stokes flows. An exception is some transient problems when small time steps are used [28].

\section{PRESSURE LAPLACIAN STABILIZATION (PLS) METHOD}

\subsection{Variational form of the mass balance equation in the PLS method}

Using the relationships (16b) we can write the second integral in Eq.(22) as

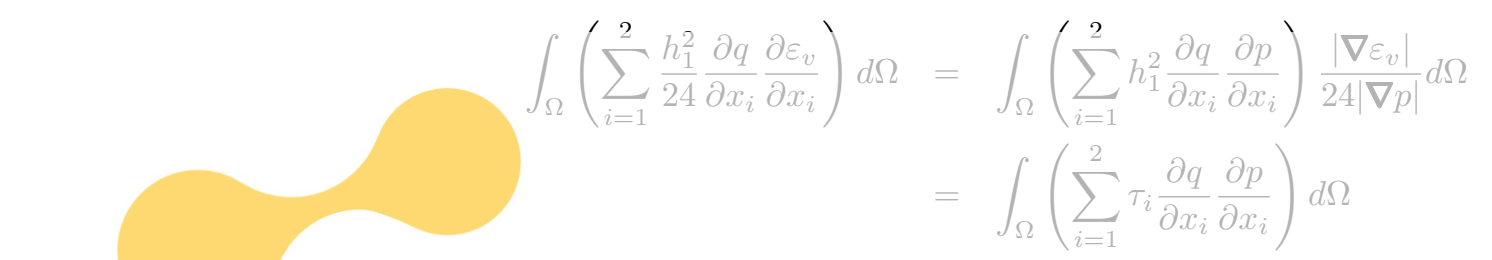

with the stabilization parameters given by
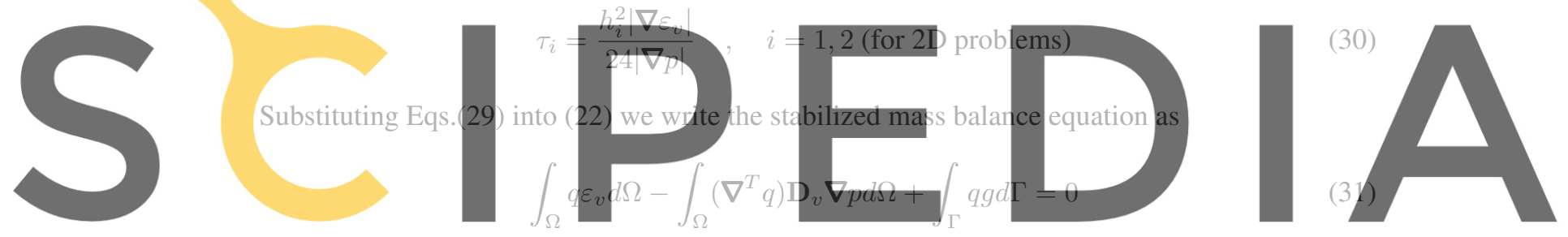

For 2D problems

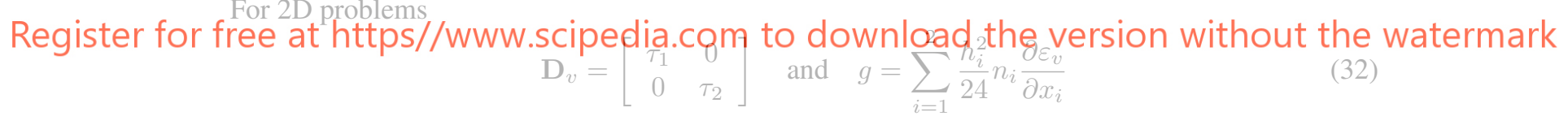

where $\mathbf{D}_{v}$ is a matrix of stabilization parameters.

\subsection{Computation of the stabilization parameters in the PLS method}

From Eq.(24) we deduce (neglecting space variations of the viscosity)

$$
\frac{2}{3} \mu\left|\nabla \varepsilon_{v}\right|=\left|\mathbf{r}_{m}\right|
$$

From the first order FIC mass balance equation (12) (with the negative sign) we deduce

$$
\frac{1}{2} h_{\xi}\left|\boldsymbol{\nabla} \varepsilon_{v}\right|=\varepsilon_{v}
$$

where $h_{\xi}$ is the projection of $\mathbf{h}$ along the gradient of $\varepsilon_{v}$, i.e.

$$
h_{\xi}=\frac{h_{i}}{\left|\boldsymbol{\nabla} \varepsilon_{v}\right|} \frac{\partial \varepsilon_{v}}{\partial x_{i}}=\frac{\mathbf{h}^{T} \boldsymbol{\nabla} \varepsilon_{v}}{\left|\boldsymbol{\nabla} \varepsilon_{v}\right|}
$$

Eqs.(33) and (34) are consistently modified as follows

$$
\frac{2}{3} \mu|\mathbf{v}|\left|\nabla \varepsilon_{v}\right|=|\mathbf{v}|\left|\mathbf{r}_{m}\right|
$$




$$
\frac{1}{2} p h_{\xi}\left|\nabla \varepsilon_{v}\right|=p \varepsilon_{v}
$$

In Eq.(36) $\mathbf{v}$ is the velocity vector.

The r.h.s. of Eqs.(36) and (37) represents the power of the residual forces in the momentum equations and of the volumetric strain rate, respectively. Note that the product $p h_{\xi}$ in Eq.(37) is always positive, as $p \varepsilon_{v} \geq 0$ (see Eq.(14)). Positiveness of $p \varepsilon_{v}$ pointwise is however ensured in the computation by taking the modulus of this product in the subsequent expressions.

From Eqs.(36) and (37) we deduce

$$
\left|\boldsymbol{\nabla} \varepsilon_{v}\right|=\frac{\left|p \varepsilon_{v}\right|+|\mathbf{v}|\left|\mathbf{r}_{m}\right|}{\frac{1}{2}\left|p h_{\xi}\right|+\frac{2}{3} \mu|\mathbf{v}|}
$$

Substituting Eq.(38) into (30) gives the expression for the stabilization parameters as

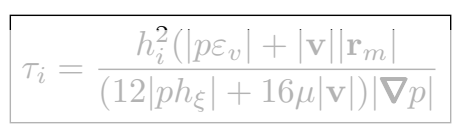

The expression for $\tau_{i}$ in Eq.(39) will vanish for values of $v_{i}$ and $p$ satisfying exactly the incompressibility equation $\left(\varepsilon_{v}=0\right)$ and the momentum equations $\left(\mathbf{r}_{m}=0\right)$. Clearly for the discrete problem, the stabilization parameters depend on the numerical errors in the approximation for $\varepsilon_{v}$ and $\mathbf{r}_{m}$. In practice, it is advisable to choose a cut-off value for the lower and upper bounds for $\tau_{i}$ avoiding very small or too large values of the stabilization parameter (for instance in zones where $\nabla p$ is smali). In the examples shown in the paper we have choson the fothewing limiting band:

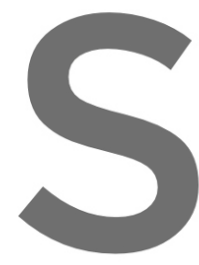
$10^{-8} \leq \tau_{i} \leq 10^{5}$.

Remark 1. Using
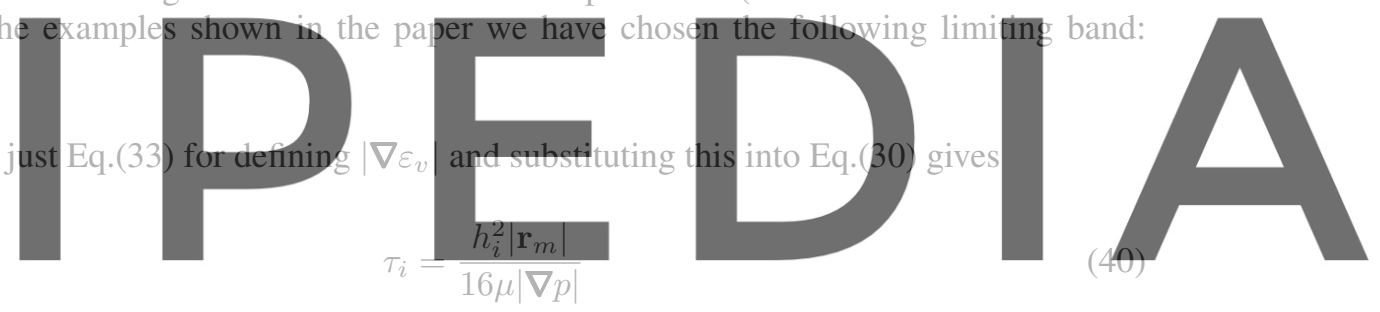

Register for free at https//www.scipedia.com to download the version without the watermark In absence of body forces and assuming steady state conditions and a linear FE approximation, then $\left|\mathbf{r}_{m}\right|=|\nabla p|$ and

$$
\tau_{i}=\frac{h_{i}^{2}}{16 \mu}
$$

which coincides with Eq.(27) deduced for the GLS method. The dimension of $\tau_{i}$ is $\frac{s \times m^{3}}{k q}$. The expression for $\tau_{i}$ in Eq.(41) is typically found in the stabilized FEM literature for Stokes flow $[1,2,16],[22]-[28]$.

Remark 2. Other residual-based expressions for the stabilization parameters $\tau_{i}$ in the PLS method can be found. For instance, two alternative expressions for $\tau_{i}$ are

$$
\tau_{i}=\frac{h_{i}^{2}}{|\nabla p|} \frac{\left(\rho|\mathbf{v}|+\frac{\rho\left|h_{\xi}\right|}{2 \Delta t}\right)\left|\varepsilon_{v}\right|+\left|\mathbf{r}_{m}\right|}{\left(12 \rho\left|h_{\xi} \mathbf{v}\right|+6 \rho \frac{h_{\xi}^{2}}{\Delta t}+16 \mu\right)}
$$

and

$$
\tau_{i}=h_{i}^{2}\left[\frac{\rho\left|h_{\xi} \mathbf{v}\right|+\mu}{24 \rho\left|h_{\xi} \mathbf{v}\right|\left|\frac{p}{\varepsilon_{v}}\right|+16 \mu^{2} \frac{\left|\nabla_{p}\right|}{\left|\mathbf{r}_{m}\right|}}\right]
$$

The derivation of above expressions can be found in [46]. 


\subsection{PLS boundary stabilization term}

From the relationships in Eq.(16b) we express the boundary term $g$ of Eq.(32) as

$$
g=\sum_{i=1}^{2} \frac{h_{i}^{2}}{24} n_{i} \frac{\partial \varepsilon_{v}}{\partial x_{i}}=\sum_{i=1}^{2} \frac{h_{i}^{2}}{24} n_{i} \frac{\left|\boldsymbol{\nabla} \varepsilon_{v}\right|}{|\boldsymbol{\nabla} p|} \frac{\partial p}{\partial x_{i}}=\sum_{i=1}^{2} \tau_{i} n_{i} \frac{\partial p}{\partial x_{i}}
$$

The boundary integral in Eq.(31) can therefore be expressed in terms of the pressure gradient components using Eq.(43) as

$$
\int_{\Gamma} q\left(\sum_{i=1}^{2} \tau_{i} n_{i} \frac{\partial p}{\partial x_{i}}\right) d \Gamma
$$

where all the terms within the integral are computed at the boundary $\Gamma$.

Remark 3. For $h_{i}=h_{j}=h$ then $\tau_{i}=\tau$. In this case, the boundary integral (46) can be expressed as

$$
\int_{\Gamma} q \tau \frac{\partial p}{\partial n} d \Gamma
$$

where $\frac{\partial p}{\partial n}=n_{i} \frac{\partial p}{\partial x_{i}}$ is the gradient of the pressure along the direction normal to the boundary.

We note that all the expressions for $\tau_{i}$ given in the previous equations are "solution dependent". The nonlinear definition of the stabilization parameters can be useful for overcoming the limitations of the standard definitions of $\tau_{\imath}$. A recent evidence of the usefulness of solution-dependent

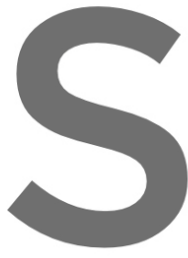

stabilization param
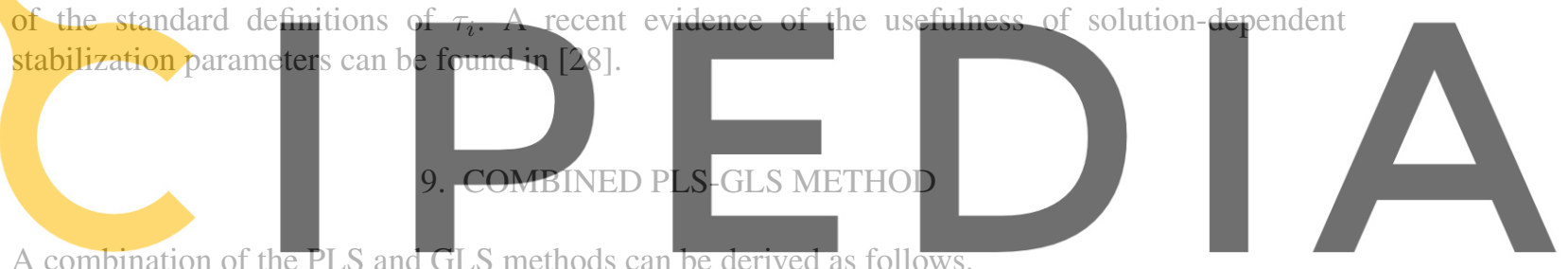

From Eqs.(16b) and (24) the following equalities hold

Register for free at https//www.scipedia.com to download the version without the watermark

$$
\begin{aligned}
& \frac{p}{\varepsilon_{v}} \frac{\partial \varepsilon_{v}}{\partial x_{i}}=\frac{\partial p}{\partial x_{i}} \\
& \frac{2}{3} \mu \frac{\partial \varepsilon_{v}}{\partial x_{i}}=r_{m_{i}}
\end{aligned}
$$

From these equations we deduce

$$
\frac{\partial \varepsilon_{v}}{\partial x_{i}}=\frac{\frac{\partial p}{\partial x_{i}}+r_{m_{i}}}{\left(\frac{p}{\varepsilon_{v}}+\frac{2}{3} \mu\right)}
$$

Substituting Eq.(48) into (22) gives

$$
\int_{\Omega} q \varepsilon_{v} d \Omega-\int_{\Omega} \sum_{i=1}^{2} \tau_{i} \frac{\partial q}{\partial x_{i}}\left(\frac{\partial p}{\partial x_{i}}+r_{m_{i}}\right) d \Omega+\int_{\Gamma} \sum_{i=1}^{2} \tau_{i} n_{i}\left(\frac{\partial p}{\partial x_{i}}+r_{m_{i}}\right) d \Gamma=0
$$

with

$$
\tau_{i}=\frac{h_{i}^{2}}{24\left(\frac{p}{\varepsilon_{v}}+\frac{2}{3} \mu\right)}
$$

The variational form (49) yields a stabilized method which combines the best features of the PLS and GLS techniques of previous sections. 


\section{COMBINED PENALTY-GLS AND PENALTY-PLS METHODS}

The penalty method of Section 6 can be combined with the GLS and PLS procedures of Sections 7 and 8 as follows.

\subsection{Combined penalty-GLS method}

Combining Eqs.(20) and (26) we can find

$$
\int_{\Omega} q \varepsilon_{v} d \Omega-\int_{\Omega}\left(\frac{\beta}{\alpha} q p+(1-\beta) \sum_{i=1}^{2} \tau_{i} \frac{\partial q}{\partial x_{i}} r_{m_{i}}\right) d \Omega+(1-\beta) \int_{\Gamma} q\left(\sum_{i=1}^{2} \tau_{i} n_{i} r_{m_{i}}\right) d \Gamma=0
$$

where $\beta$ is a parameter such that $0 \leq \beta \leq 1$.

The variational form (51) yields a stabilized method which combines the key ingredients of the penalty and GLS techniques.

\subsection{Combined penalty-PLS method}

From Eqs.(20) and (31) we can write

$$
\int_{\Omega} q \varepsilon_{v} d \Omega-\int_{\Omega}\left[\frac{\beta}{\alpha} q p+(1-\beta)\left(\boldsymbol{\nabla}^{T} q\right) \mathbf{D}_{v} \boldsymbol{\nabla} p\right] d \Omega+(1-\beta) \int_{\Gamma} q g d \Gamma=0
$$

where again $0 \leq \beta \leq 1$

The variational form (52) yields a stabilized method that combines the best features of the penalty and the PLS methods.

\section{PRESSURE-GRADIENT PROJECTION (PGP) FORMULATION}

An alternative stabilized formulation can be derived from the higher order FIC equations by introducing the so-called pressure-gradient projection variables. The resulting stabilized mass balance equations can be derived is a number of ways [1, 2, 15, 16, 23]. Here we show how a PGP (for pressure-gradient projection) method can be readily obtained following the higher order FIC approach.

From the momentum equations it can be found

$$
\frac{h_{i}^{2}}{24} \frac{\partial \varepsilon_{v}}{\partial x_{i}}=\tau_{i} r_{m_{i}}
$$

where $r_{m_{i}}$ is defined in Eq.(25a). An expression for the stabilization parameter $\tau_{i}$ is [1, 2, 15, 16, 23]

$$
\tau_{i}=\frac{h_{i}^{2}}{24}\left[\frac{\rho l^{2}}{4 \Delta t}+\frac{2 \mu}{3}\right]^{-1}
$$

and $l$ is a typical grid distance. The expression for $\tau_{i}$ of Eq.(54) can be obtained as a particular case of Eq.(42a).

For relatively fine grids the numerical solution is insensitive to the values of $h_{i}:=\alpha_{i} l$ [30]. In [46] we found that good results are obtained for the range of values of $h_{i}$ such that $\sqrt{2} \leq \alpha_{i} \leq \sqrt{6}$, where $l$ is a typical grid distance.

For the steady state problems solved in this work the form of $\tau_{i}$ of Eq.(27) is used with $\alpha_{i}=\alpha=\sqrt{6}$.

Note that the stabilization parameters in the PGP method are constant for each element. This is an important difference versus the PLS and penalty formulations of previous sections, where a non linear (and consistent) form for the stabilization parameters is used. 
In the standard PGP method the momentum residuals $r_{m_{i}}$ are split as $r_{m_{i}}:=\frac{\partial p}{\partial x_{i}}+\pi_{i}$ where $\pi_{i}$ are the so-called pressure-gradient projection variables $[15,16]$. In our work we use a slight different approach and split the momentum equations as

$$
r_{m_{i}}:=\frac{\partial p}{\partial x_{i}}+\frac{1}{\tau_{i}} \pi_{i}
$$

where

$$
\pi_{i}=\tau_{i}\left(-\rho \frac{\partial v_{i}}{\partial t}+\frac{\partial s_{i j}}{\partial x_{j}}+b_{i}\right)
$$

is the $i$ th pressure-gradient projection weighted by the $i$ th stabilization parameter. The $\pi_{i}$ 's are now taken as additional variables which are discretized with the standard FEM in the same manner as for the pressure.

The split of Eq.(55) ensures that the term $\frac{1}{\tau_{i}} \pi_{i}$ is discontinuous between adjacent elements after discretization. This is essential for accurately capturing high discontinuous pressure gradient jumps typical of fluids with heterogeneous physical properties (either the viscosity or the pressure) $[39,44,45]$. In this manner the term $\frac{1}{\tau_{i}} \pi_{i}$ can match the discrete pressure gradient term $\frac{\partial p}{\partial x_{i}}$ which is naturally discontinuous between elements for a linear approximation of the pressure.

Substituting Eq.(53) into the second and third integral of Eq.(22) and using (55) gives (for 2D problems)

$$
\int_{\Omega} q \varepsilon_{v} d \Omega-\int_{\Omega} \sum_{i=1}^{2} \frac{\partial q}{\partial x_{i}}\left(\tau_{i} \frac{\partial p}{\partial x_{i}}+\pi_{i}\right) d \Omega+\int_{\Gamma} q \sum_{i=1}^{2} n_{i}\left(\tau_{i} \frac{\partial p}{\partial x_{i}}+\pi_{i}\right) d \Gamma=0
$$

The boundary integral in Eq.(57) is typically neglected in PGP formulations and will be disregarded from here onward.

The following addition equations are introduced for computing the pressure gradient projection variables $\pi_{i}$

$$
\int_{\Omega} \sum_{i=1}^{2} \bar{w}_{i}\left(\frac{\partial p}{\partial x_{i}}+\frac{1}{\tau_{i}} \pi_{i}\right) d \Omega=0
$$

where $\bar{w}_{i}=q$ is usually taken.

Recall that the term $\frac{\partial p}{\partial x_{i}}+\frac{1}{\tau_{i}} \pi_{i}$ (no sum in $i$ ) is an alternative expression for the momentum residual equation (see Eq.(55)). This term is enforced to vanish in an average sense via Eq.(58).

\section{ORTHOGONAL SUB-SCALES (OSS) FORMULATION}

The OSS method can be readily derived by writing the momentum residuals as

$$
r_{m_{i}}=\left(\bar{r}_{m_{i}}+\pi_{i}\right)
$$

where $\bar{r}_{m_{i}}$ are the discrete residuals of the momentum equations and $\pi_{i}$ are additional variables that are now interpreted as the projection of the discrete momentum residuals into the velocity space (without boundary conditions) [17, 20,21].

The variational form for the incompressibility equations is obtained by substituting Eq.(59) into (26). This gives (neglecting the boundary terms)

$$
\int_{\Omega} q \varepsilon_{v} d \Omega-\int_{\Omega} \sum_{i=1}^{2} \frac{\partial q}{\partial x_{i}} \tau_{i}\left(\bar{r}_{m_{i}}+\pi_{i}\right) d \Omega=0
$$

The $\pi_{i}$ variables are computed by the following additional equations

$$
\int_{\Omega} \sum_{i=1}^{2} \bar{w}_{i}\left(\bar{r}_{m_{i}}+\pi_{i}\right) d \Omega=0
$$


with $\bar{w}_{i}=q$. Eq.(61) enforces the consistency of the method in an average sense.

The stabilization parameters $\tau_{i}$ are computed as in the PGP method of previous section.

Remark 4 . For linear FE interpolations the term $\bar{r}_{m_{i}}$ is simply

$$
\bar{r}_{m_{i}}=-\rho \frac{\partial \bar{v}_{i}}{\partial t}+\frac{\partial \bar{p}}{\partial x_{i}}+b_{i}
$$

where $\bar{v}_{i}$ and $\bar{p}$ are the approximate FE values for the velocities and the pressure.

\section{PLS $+\pi$ METHOD}

The PLS method presented in Section 10 can be substantially enhanced if the momentum residuals appearing in the expression for the stabilization parameters $\tau_{i}$ are computed using Eq.(59). The resulting expression for $\tau_{i}$ is

$$
\tau_{i}=\frac{h_{i}^{2}\left(\left|p \varepsilon_{v}\right|+|\mathbf{v}|\left|\overline{\mathbf{r}}_{m}+\boldsymbol{\pi}\right|\right)}{\left(12\left|p h_{\xi}\right|+16 \mu|\mathbf{v}|\right)|\boldsymbol{\nabla} p|}
$$

This $\boldsymbol{\pi}$ variables are computed via Eq.(61) following the discretization procedure described in the next section.

The expression for $\tau_{i}$ of Eq.(63) increases the efficiency and accuracy of the PLS method (see Example 17.5).

\section{FINITE ELEMENT DISCRETIZATION}

\subsection{Discretized equations}

The domain $\Omega$ is discretized with a mesh of triangles or quadrilaterals (for $2 \mathrm{D}$ ) and tetrahedra or hexahedra (for 3D).

For the penalty, GLS and PLS formulations, the velocities and the pressure are interpolated over each element using the same approximation as (for 3D problems)

$$
\mathbf{v}=\left\{\begin{array}{l}
v_{1} \\
v_{2} \\
v_{3}
\end{array}\right\}=\sum_{j=1}^{n} \mathbf{N}_{j} \overline{\mathbf{v}}_{j} \quad, \quad p=\sum_{j=1}^{n} N_{j} \bar{p}_{j}
$$

For the PGP and OSS formulations the $\boldsymbol{\pi}_{i}$ variables are also interpolated using the shape functions $N_{i}$ as

$$
\boldsymbol{\pi}=\left\{\begin{array}{l}
\pi_{1} \\
\pi_{2} \\
\pi_{3}
\end{array}\right\}=\sum_{j=1}^{n} \mathbf{N}_{j} \overline{\boldsymbol{\pi}}^{j}
$$

In the above equations $\mathbf{N}_{j}=N_{j} \mathbf{I}_{3}, N_{j}$ is the standard shape function for node $j, \mathbf{I}_{3}$ is the $3 \times 3$ unit matrix, $n$ is the number of nodes in the element (i.e. $n=3 / 4$ for linear triangles/tetrahedra) and $\overline{\mathbf{v}}_{j}, \bar{p}_{j}$ and $\overline{\boldsymbol{\pi}}^{j}$ are the values of the velocity vector, the pressure and the $\boldsymbol{\pi}$ variables vector at node $j$, respectively. Indeed, any other FE approximation for $\mathbf{v}, p$ and $\boldsymbol{\pi}$ can be used.

Substituting the approximations (64) and (65) into the weak form of the momentum equations (Eq.(10)) and in the adequate variational expression for the incompressibility equation, gives the following global system of equations (for all the methods considered in the paper)

$$
\overline{\mathbf{M}} \dot{\bar{a}}+\mathbf{H} \overline{\mathbf{a}}=\mathbf{f}
$$

where $\dot{\overline{\mathbf{a}}}=\frac{\mathrm{d}}{\mathrm{dt}} \overline{\mathbf{a}}$ and the different matrices and vectors for the different stabilized FE methods are 


\section{Penalty, GLS, PLS and combined methods}

$$
\overline{\mathbf{a}}=\left\{\begin{array}{c}
\overline{\mathbf{v}} \\
\overline{\mathbf{p}}
\end{array}\right\} \quad, \quad \overline{\mathbf{M}}=\left[\begin{array}{ll}
\mathbf{M} & \mathbf{0} \\
\hat{\mathbf{M}} & \mathbf{0}
\end{array}\right] \quad, \quad \mathbf{H}=\left[\begin{array}{cc}
\mathbf{K} & \mathbf{Q} \\
\left(\mathbf{Q}^{T}+\mathbf{R}\right) & \mathbf{S}
\end{array}\right] \quad, \quad \mathbf{f}=\left\{\begin{array}{l}
\mathbf{f}_{v} \\
\mathbf{f}_{p}
\end{array}\right\}
$$

where

$$
\begin{aligned}
& \text { Penalty method: } \quad \mathbf{S}=\mathbf{P} \quad, \quad \mathbf{R}=\hat{\mathbf{M}}=\mathbf{0} \quad, \quad \mathbf{f}_{p}=\mathbf{0} \\
& \text { GLS method: } \quad \mathbf{S}=-\mathbf{L}+\mathbf{B} \\
& \text { PLS method: } \quad \mathbf{S}=-\mathbf{L}+\mathbf{B} \quad, \quad \mathbf{R}=\hat{\mathbf{M}}=\mathbf{0} \quad, \quad \mathbf{f}_{p}=\mathbf{0} \\
& \text { PLS-GLS method: } \quad \mathbf{S}=-2 \mathbf{L}+\mathbf{B} \\
& \text { Penalty-GLS method: } \quad \mathbf{S}=\beta \mathbf{P}+(1-\beta)(-\mathbf{L}+\mathbf{B}) \quad, \quad \mathbf{R}=(1-\beta) \mathbf{R} \\
& \text { Penalty-PLS method : } \quad \mathbf{S}=\beta \mathbf{P}+(1-\beta)(-\mathbf{L}+\mathbf{B}) \quad, \quad \mathbf{R}=\hat{\mathbf{M}}=\mathbf{0} \quad, \quad \mathbf{f}_{p}=\mathbf{0}
\end{aligned}
$$

\section{PGP method}

$$
\overline{\mathbf{a}}=\left\{\begin{array}{c}
\overline{\mathbf{v}} \\
\overline{\mathbf{p}} \\
\overline{\boldsymbol{\pi}}
\end{array}\right\} \quad, \quad \overline{\mathbf{M}}=\left[\begin{array}{ccc}
\mathbf{M} & \mathbf{0} & \mathbf{0} \\
\mathbf{0} & \mathbf{0} & \mathbf{0} \\
\mathbf{0} & \mathbf{0} & \mathbf{0}
\end{array}\right] \quad, \quad \mathbf{H}=\left[\begin{array}{ccc}
\mathbf{K} & \mathbf{Q} & \mathbf{0} \\
\mathbf{Q}^{T} & -\mathbf{L} & -\mathbf{C} \\
\mathbf{0} & -\mathbf{C}^{T} & -\mathbf{T}
\end{array}\right] \quad, \quad \mathbf{f}=\left\{\begin{array}{c}
\mathbf{f}_{v} \\
\mathbf{0} \\
\mathbf{0}
\end{array}\right\}
$$

\section{OSS method}

$\overline{\mathbf{a}}$ and $\mathbf{H}$ as in Eq.(68)

$$
\overline{\mathbf{M}}=\left[\begin{array}{ccc}
\mathbf{M} & \mathbf{0} & \mathbf{0} \\
\overline{\mathbf{M}}_{p} & \mathbf{0} & \mathbf{0} \\
\mathbf{M}_{\pi} & \mathbf{0} & \mathbf{0}
\end{array}\right] \quad, \quad \mathbf{f}=\left\{\begin{array}{c}
\mathbf{f}_{v} \\
\mathbf{f}_{p} \\
\mathbf{f}_{\pi}
\end{array}\right\} \quad \text { with } \quad \mathbf{f}_{\pi}=\left\{\begin{array}{c}
\mathbf{f}_{\pi_{1}} \\
\mathbf{f}_{\pi_{2}} \\
\mathbf{f}_{\pi_{3}}
\end{array}\right\}
$$

The matrices and vectors in Eqs.(67)-(69) are formed by assembling the element contributions given in Box 1 for 3D problems.

Remark 5. For linear triangles, matrices $\mathbf{M}, \mathbf{P}$ and $\mathbf{T}$ are computed with a three point Gauss quadrature. The rest of the matrices and vectors in Box 1 are computed with just a one-point quadrature. A higher order quadrature might be required in some cases for integrating more accurately the non linear terms involving the stabilization parameters $\tau_{i}$.

Remark 6. The contribution of the stabilization terms to the stiffness matrix $\mathbf{K}$ which are typical in the standard GLS formulation are not taken into account in this work. These terms are irrelevant for the analysis of the steady-state Stokes problems presented in the paper.

\subsection{Solution schemes for penalty, GLS, PLS and combined methods}

For penalty, GLS, PLS and combined methods, a monolithic transient solution of Eq.(66) can be found using the following iterative scheme

$$
{ }^{j+1} \overline{\mathbf{a}}^{n+1}=\left[{ }^{j} \overline{\mathbf{H}}^{n+1}\right]^{-1}\left(\mathbf{f}+\frac{1}{\Delta t} \overline{\mathbf{M}} \mathbf{a}^{n}\right)
$$

with

$$
\overline{\mathbf{H}}=\mathbf{H}+\frac{1}{\Delta t} \overline{\mathbf{M}}
$$

In Eq.(70a) $(\cdot)^{n}$ and $(\cdot)^{n+1}$ denote values at times $t$ and $t+\Delta t$, respectively while the upper left index $j$ denotes the iteration number; i.e. ${ }^{j}(\cdot)^{n+1}$ denotes values at time $t+\Delta t$ and the $j$ th iteration. 


$$
\mathbf{M}_{i j}^{e}=\int_{\Omega^{e}} \rho \mathbf{N}_{i}^{T} \mathbf{N}_{j} d \Omega \quad, \quad \mathbf{K}_{i j}^{e}=\int_{\Omega^{e}} \mathbf{G}_{i}^{T} \mathbf{D} \mathbf{G}_{j} d \Omega \quad, \quad \mathbf{Q}_{i j}^{e}=\int_{\Omega^{e}} \mathbf{G}_{i}^{T} \mathbf{m} N_{j} d \Omega
$$

with

$$
\mathbf{G}_{i}=\left[\begin{array}{ccc}
\frac{\partial N_{i}}{\partial x_{1}} & 0 & 0 \\
0 & \frac{\partial N_{i}}{\partial x_{2}} & 0 \\
0 & 0 & \frac{\partial N_{i}}{\partial x_{3}} \\
\frac{\partial N_{i}}{\partial x_{2}} & \frac{\partial N_{i}}{\partial x_{1}} & 0 \\
\frac{\partial N_{i}}{\partial x_{3}} & 0 & \frac{\partial N_{i}}{\partial x_{1}} \\
P_{i j}^{e}=\int_{\Omega} \frac{\partial N_{i}}{\alpha} & \frac{\partial N_{i}}{\partial x_{2}}
\end{array}\right], \quad \mathbf{m}=[1,1,1,0,0,0]^{T} \quad, \quad \mathbf{D}=\mu\left[\begin{array}{cc}
2 \mathbf{I}_{3} & 0 \\
0 & \mathbf{I}_{3}
\end{array}\right]
$$

$\mathbf{M}_{p}^{e}$ as $\hat{\mathbf{M}}^{e}$ neglecting the boundary term.

$$
\begin{gathered}
\mathbf{R}_{i j}^{e}=-\int_{\Omega^{e}}\left(\boldsymbol{\nabla}^{T} N_{i}\right)[\tau] \mathbf{L}^{T} \mathbf{D} \mathbf{G}_{j} d \Omega+\int_{\Gamma^{e}} N_{i} \boldsymbol{\tau}_{n}^{T}\left(\mathbf{L}^{T} \mathbf{D} \mathbf{G}_{j}\right) d \Gamma \\
\boldsymbol{\tau}=\left[\tau_{1}, \tau_{2}, \tau_{3}\right]^{T},[\tau]=\left[\begin{array}{ccc}
\tau_{1} & 0 & 0 \\
0 & \tau_{2} & 0 \\
0 & 0 & \tau_{3}
\end{array}\right] \quad, \quad \boldsymbol{\tau}_{n}=[\tau] \mathbf{n} \\
\mathbf{P G P}: \quad \mathbf{C}_{i j}^{e}=\int_{\Omega^{e}} \mathbf{m}^{T} \mathbf{G}_{i} \mathbf{N}_{j} d \Omega \quad, \quad \mathbf{T}_{i j}^{e}=\int_{\Omega^{e}} \mathbf{N}_{i}[\tau]^{-1} \mathbf{N}_{j} d \Omega \\
\mathbf{O S S :} \quad \mathbf{C}_{i j}^{e}=\int_{\Omega^{e}} \mathbf{m}^{T} \mathbf{G}_{i}[\tau] \mathbf{N}_{j} d \Omega, \quad \mathbf{T}_{i j}^{e}=\int_{\Omega^{e}} \mathbf{N}_{i}[\tau] \mathbf{N}_{j} d \Omega \\
f_{p_{i}}^{e}=\int_{\Omega^{e}}\left(\sum_{j=1}^{3} \tau_{j} \frac{\partial N_{i}}{\partial x_{j}} b_{j}\right) d \Omega-\int_{\Omega^{e}} N_{i} \mathbf{b} d \Omega+\int_{\Gamma_{t}^{e}} N_{i} \mathbf{t}^{p} d \Gamma \quad, \quad i, j=1,2,3
\end{gathered}
$$

$\bar{f}_{p_{i}}$ as $f_{p_{i}}$ neglecting the boundary term.

$\mathbf{I}_{3}: 3 \times 3$ unit matrix $\quad, \quad \mathbf{b}=\left[b_{1}, b_{2}, b_{3}\right]^{T} \quad, \quad \mathbf{t}^{p}=\left[t_{1}^{p}, t_{2}^{p}, t_{3}^{p}\right]^{T}$

$\Gamma_{t}^{e}$ : boundary of element $e$ coincident with the external Neuman boundary

Box 1. Element expressions for the matrices and vectors in Eqs.(67)-(69) for 3D problems. The expression for $\tau_{i}$ changes for the different stabilized methods

For the steady state problems solved in this work we have found the velocity and pressure variables simultaneously by inverting the system

$$
\mathbf{H a}=\mathbf{f}
$$


Clearly, for the PLS method the solution of Eq.(71) must be found iteratively, as the stabilization parameters are a function of the velocity and the pressure. A simple direct iteration scheme gives

$$
{ }^{j+1} \overline{\mathbf{a}}=\left[{ }^{j} \mathbf{H}\right]^{-1} \mathbf{f}
$$

For transient problems, an implicit segregated approach has typically more advantages. For instante, the following iterative scheme can be used for computing $\overline{\mathbf{v}}$ and $\overline{\mathbf{p}}$ in time as

Step 1

$$
{ }^{j+1} \overline{\mathbf{v}}^{n+1}=\overline{\mathbf{v}}^{n}+\left[\frac{1}{\Delta t} \mathbf{M}+\mathbf{K}\right]^{-1}\left[\mathbf{f}_{v}+\mathbf{Q}^{j} \overline{\mathbf{p}}^{n+1}\right]
$$

Step 2

$$
{ }^{j+1} \overline{\mathbf{p}}^{n+1}=\mathbf{S}^{-1}\left[\mathbf{f}_{p}-\left(\mathbf{Q}^{T}+\mathbf{R}\right)^{j+1} \overline{\mathbf{v}}^{n+1}-\frac{1}{\Delta t} \hat{\mathbf{M}}\left(\overline{\mathbf{v}}^{n+1}-\overline{\mathbf{v}}^{n}\right)\right]
$$

It is important to note that neglecting matrix B in Eq.(73b) for the GLS and PLS methods would require prescribing the pressure at some point of the domain for inverting the Laplace matrix $\mathbf{L}$. As mentioned earlier, the typical option of making $p=0$ at a free boundary introduces an error in the mass conservation equation leading to mass losses for viscous free surface flow problems [43]. The presence of the boundary matrix B in Eq.(73b) avoids the need for prescribing the pressure at the boundary in those cases.

\subsection{Solution scheme for PGP and OSS methods}

The solution of Eqs.(66) for the PGP and OSS methods is typically performed via an iterative staggered scheme.

The $\overline{\boldsymbol{\pi}}$ nodal variables can be eliminated from Eqs.(66) as follows

$$
\begin{array}{ll}
\text { PGP method: } & \overline{\boldsymbol{\pi}}=-\mathbf{T}^{-1} \mathbf{C}^{T} \overline{\mathbf{p}} \\
\text { OSS method: } & \overline{\boldsymbol{\pi}}=-\mathbf{T}^{-1}\left(\mathbf{M}_{\pi} \dot{\overline{\mathbf{v}}}+\mathbf{C}^{\mathbf{T}} \overline{\mathbf{p}}-\mathbf{f}_{\pi}\right)
\end{array}
$$

Substituting $\overline{\boldsymbol{\pi}}$ from Eqs.(74) into the second row of Eqs.(66) yields the following system of two equations for $\overline{\mathbf{v}}$ and $\overline{\mathbf{p}}$

$$
\begin{aligned}
& \text { PGP method: } \quad \begin{array}{ll}
\mathbf{M} \dot{\overline{\mathbf{v}}}+\mathbf{K} \overline{\mathbf{v}}+\mathbf{Q} \overline{\mathbf{p}}=\mathbf{f}_{v} \\
\mathbf{Q} \overline{\mathbf{v}}-(\mathbf{L}-\hat{\mathbf{L}}) \overline{\mathbf{p}}=\mathbf{0}
\end{array} \\
& \text { OSS method: } \quad \begin{array}{l}
\mathbf{M} \dot{\overline{\mathbf{v}}}+\mathbf{K} \overline{\mathbf{v}}+\mathbf{Q} \overline{\mathbf{p}}=\mathbf{f}_{v} \\
\left(\overline{\mathbf{M}}_{p}-\mathbf{C} \mathbf{T}^{-1} \mathbf{M}_{\pi}\right) \dot{\overline{\mathbf{v}}}-(\mathbf{L}-\hat{\mathbf{L}}) \overline{\mathbf{p}}=\overline{\mathbf{f}}_{p}-\mathbf{T}^{-1} \mathbf{f}_{\pi}
\end{array}
\end{aligned}
$$

In Eqs.(75) $\hat{\mathbf{L}}=\mathbf{C T}^{-1} \mathbf{C}^{T}$ is the discrete pressure Laplace matrix. This matrix has a wider bandwidth than the Laplace pressure matrix $\mathbf{L}$ in Eqs.(67b). The difference between $\mathbf{L}$ and $\hat{\mathbf{L}}$ provides the necessary stabilization for the accurate solution of Eqs.(75).

The system of equations (75) can be solved in time with an iterative segregated scheme.

For the steady state case the solution for the velocity and pressure is found simultaneously by Eq.(71) with

$$
\mathbf{H}=\left[\begin{array}{cc}
\mathbf{K} & \mathbf{Q} \\
\mathbf{Q}^{T} & (\hat{\mathbf{L}}-\mathbf{L})
\end{array}\right] \quad, \quad \mathbf{a}=\left\{\begin{array}{c}
\overline{\mathbf{v}} \\
\overline{\mathbf{p}}
\end{array}\right\} \quad, \quad \mathbf{f}=\left\{\begin{array}{c}
\mathbf{f}_{v} \\
\hat{\mathbf{f}}_{p}
\end{array}\right\}
$$

where $\hat{\mathbf{f}}_{p}=\mathbf{0}$ for the PGP method and $\hat{\mathbf{f}}_{p}=\overline{\mathbf{f}}_{p}-\mathbf{T}^{-1} \mathbf{f}_{\pi}$ for the OSS method. 
Remark 7. The elimination of the $\boldsymbol{\pi}$ variables via Eqs.(74) can be simplified by using a diagonal form of matrix $\mathbf{T}$ obtained as $\mathbf{T}_{d}=\operatorname{diag}(\mathbf{T})$. This, however, does not affect the bandwith of matrix $\hat{\mathbf{L}}$.

Remark 8. The bracketed matrix multiplying $\dot{\overline{\mathbf{v}}}$ in the second of Eqs.(75b) vanishes for the case of constant density.

\section{DEFINITION OF THE CHARACTERISTIC LENGTHS}

In our work we have used the following definition for $h_{i}$ for all the stabilized methods studied

$$
h_{i}=\max \left[\mathbf{l}_{j}^{T} \mathbf{a}_{j}\right] \quad, \quad j=1, n_{s}
$$

with $\mathbf{a}_{1}=[1,0]^{T}$ and $\mathbf{a}_{2}=[0,1]^{T}$ for 2D problems, $\mathbf{l}_{i}$ are the vectors along the sides of the element and $n_{s}$ is the number of sides ( $n_{s}=3$ for triangles). For instance, for side 1 of a triangle linking nodes 2 and $3, \mathbf{l}_{i}=\left[x_{1}^{3}-x_{1}^{2}, x_{2}^{3}-x_{2}^{2}\right]^{T}$, where $x_{1}^{i}, x_{2}^{i}$ are the horizontal and vertical coordinates of node $i$.

For the examples solved in this paper results using the expression for $h_{i}$ of Eq.(77) have been found to be very similar in all cases to those using a constant value for $h_{i}$ defined as

$$
h_{i}=h^{e} \quad \text { with } h^{e}=\left[A^{e}\right]^{1 / 2}
$$

\section{SOME COMMENTS ON THE DIFFERENT STABILIZATION METHODS}

\subsection{Penalty method}

For the penalty method the system matrix $\mathbf{H}$ is symmetrical. Matrix $\mathbf{M}$ becomes ill-conditioned for very large values of the penalty parameter $\alpha$. A cut-off for $\alpha$ when the solution approaches the incompressibility limit is therefore mandatory to avoid the volumetric locking problem $[1,2]$.

\subsection{GLS method}

For the GLS method presented here matrices $\hat{\mathbf{M}}, \mathbf{R}$ and $\mathbf{B}$ are non symmetrical. Symmetry of matrices $\overline{\mathbf{M}}$ and $\mathbf{H}$ can be simply found by shifting the non symmetrical terms to the r.h.s. of Eqs.(66). This introduces a non linearity in the solution scheme.

\subsection{PLS method}

For the PLS method the boundary stabilization matrix B is non-symmetrical. Symmetry of the system matrix $\mathbf{H}$ can be recovered by shifting the boundary terms to the r.h.s. of Eq.(66). This gives

$$
\mathbf{H}=\left[\begin{array}{cc}
\mathbf{K} & \mathbf{Q} \\
\mathbf{Q}^{T} & -\mathbf{L}
\end{array}\right] \quad, \quad \mathbf{f}=\left\{\begin{array}{l}
\mathbf{f}_{v} \\
\mathbf{q}_{p}
\end{array}\right\}
$$

For 3D problems

$$
q_{p_{i}}^{e}=\int_{\Gamma^{e}} N_{i}\left(\sum_{j=1}^{3} \tau_{j} n_{j} \frac{\partial p}{\partial x_{j}}\right) d \Gamma
$$

The boundary force vector $\mathbf{q}_{p}$ is computed at each iteration as part of the iterative solution process. Preliminary experiences in applying this method show that this does not increase the total number of iterations.

Symmetry of the boundary stabilization matrix can also be recovered by defining matrix $\mathbf{B}$ as

$$
B_{i j}^{e}=\int_{\Gamma} \tau N_{i} N_{j} d \Gamma \quad \text { with } \tau=\sum_{k=1}^{3} \frac{\tau_{k} n_{k}}{p} \frac{\partial p}{\partial x_{k}}
$$


Matrix $B_{i j}^{e}$ of Eq.(80) has now the form of a boundary mass-type matrix. The price for the symmetry of $\mathbf{B}$ is the increased non linearity of the boundary stabilization parameter.

\subsection{Combined penalty-GLS and penalty-PLS methods}

The combination of the stabilization matrix emanating from the penalty method with that of the GLS method or the PLS method can be advantageous to increase the robustness of the overall stabilized formulation in problems where the GLS and PLS formulations have difficulties to obtain a stable solution throughout the analysis domain.

\subsection{PGP and OSS method}

All matrices in the PGP method are symmetrical. For the OSS method matrix $\overline{\mathbf{M}}$ is not symmetrical. Symmetry of matrix $\overline{\mathbf{M}}$ can be found by shifting the non symmetrical terms (involving time derivatives of the velocities) to the r.h.s. of Eq.(66).

\subsection{Comparison between the different stabilization methods}

1. In the PGP and OSS methods the stabilization parameter is typically taken as constant (at least for homogeneous meshes and constant viscous fluids). In the PLS method, however, the stabilization parameter $\tau_{i}$ varies as a function of the volumetric strain rate and the residual of the momentum equations.

2. In the PGP and OSS methods the amount of stabilization is variable in space. This variation is introduced by the difference between the Laplace pressure matrix $\mathbf{L}$ and the discrete pressure Laplace matrix $\hat{\mathbf{L}}$. In the PLS method (and also in the penalty method presented here) the amount of stabilization is also variable in space, but the variation is introduced by the consistent stabilization parameters.

3. The consistency in the GLS methods is guaranteed by introducing the discrete residual of the momentum equations in the stabilized mass balance equation. Consistency in the PGP and OSS methods is enforced by introducing additional equations representing the vanishing of the momentum residuals in an average sense. In the PLS method the consistency is guaranteed by the expression of the stabilization parameters which also vanish for the exact solution (i.e. for $\varepsilon_{v}=0$ and $r_{m_{i}}=0$ ).

4. The PLS method is nonlinear due to the dependence of the stabilization parameters with the volumetric strain rate, the pressure, the pressure gradient and the residual of the momentum equations. The PGP and OSS methods are non linear due to the definition of the $\pi_{i}$ variables which are a function of the pressure field. The GLS method can be considered as a linear method.

5. All methods, except the penalty method, introduce a boundary stabilization term. Accounting for this term is relevant in the GLS and PLS methods for free surface flow problems.

6. In PGP and OSS methods the boundary stabilization term is usually neglected. This simplification is acceptable on external boundaries, but cannot be neglected at internal interfaces with a jump in the physical properties.

7. PGP and OSS methods typically yield identical results for problems when the force term $b_{i}$ belongs to the space of finite element functions and linear (or bilinear) elements are used [15].

8. The PLS and GLS methods are a priori more efficient than the PGP and OSS methods as:

(i) They do not need the evaluation of an auxiliary vector field (i.e. the $\pi_{i}$ variables).

(ii) The bandwidth of the assembled equation system for the pressure is smaller due to the larger bandwidth of the discrete Laplace matrix $\hat{\mathbf{L}}$.

(iii) As a consequence of (i) and (ii) the computational cost of the PLS and GLS methods is smaller.

(iv) The PLS method can represent exactly a pressure gradient jump.

Numerical results reported in the next section and in [46] show that the PLS in many cases even more accurate than the GLS, PGP and OSS methods. 


\section{EXAMPLES OF APPLICATION}

We present a number of examples of simple steady-state Stokes flow problems. The aim is to validating and comparing the accuracy and efficiency of some of the methods presented in the paper. The methods compared are:

- PLS method of Section 8 using the expression for $\tau_{i}$ of Eq.(39). The effect of including or not the boundary integral (BI) terms of Eq.(31) has been studied.

- PLS $+\pi$ method of Section 13. This method was used just in the manufactured flow problem (Section 17.5).

- GLS method of Section 7, including and excluding the boundary integral (BI) terms of Eq.(28).

- OSS method of Section 12 with consistent and diagonal forms of matrix $\mathbf{T}$.

The problems solved are the following:

i) Hydrostatic flow problem for a single fluid in a square domain.

ii) Two-fluid hydrostatic problem in a square domain.

iii) Poiseuille flow in a trapezoidal domain.

iv) Lid driven cavity flow problem.

v) Manufactured flow problem in a trapezoidal domain.

For all problems the nodal velocities and pressures have been found simultaneously under steadystate conditions by solving Eq.(71). For the GLS and the OSS methods the solution is found in a single step. For the PLS method the direct iteration scheme of Eq.(72) is used. The first PLS (and PLS $+\pi$ ) solution is found in all cases using a constant value of $\tau_{i}=\tau=10^{-5}$. This roughly corresponds to the value of $\tau_{i}$ for the GLS and OSS methods given by Eq.(27).

The convergence of the nonlinear iterations for the PLS method is measured in the Euclidean vector norm for velocities and pressure measured as

$$
\frac{\left\|^{j} \overline{\boldsymbol{\phi}}-{ }^{j-1} \overline{\boldsymbol{\phi}}\right\|_{L^{2}}}{\|j \overline{\boldsymbol{\phi}}\|_{L^{2}}} \leq 10^{-4} \quad \text { with } \quad \boldsymbol{\phi}_{k}=\left\{\begin{array}{c}
\bar{v}_{1_{k}} \\
\bar{v}_{2_{k}} \\
\bar{p}_{k}
\end{array}\right\} \quad \text { and } \quad\|\overline{\boldsymbol{\phi}}\|_{L^{2}}=\left[\sum_{a}\left(\overline{\boldsymbol{\phi}}^{a}\right)^{2}\right]^{1 / 2}
$$

A comparison of the PLS and PGP methods for problems i, ii and iv is reported in [46].

\subsection{Hydrostatic flow problem for a single fluid in a square domain}

We solve for the pressure distribution in a square container filled with water. The body forces are $b_{1}=b_{2}=\rho g$ with values of the density and gravity constant equal to $\rho=1000 \mathrm{Kg} / \mathrm{m}^{3}$ and $g=-10$ $\mathrm{m} / \mathrm{s}^{2}$, respectively. The viscosity is $\mu=10^{-3} \mathrm{Ns} / \mathrm{m}^{2}$. The normal velocity has been prescribed to zero at the bottom line and the two vertical walls. The nodes on the top surface are allowed to move freely. The solution for this simple problem is $\mathbf{v}=\mathbf{0}$ an hydrostatic distribution of the pressure which is independent of the fluid viscosity. The problem is solved with a $2 \times 10 \times 10$ mesh of 3noded triangles.

Figure $2 \mathrm{a}$ shows the pressure distribution obtained all methods. A converged solution which approximates practically the exact hydrostatic distribution is found with the PLS method in just two iterations.

\subsection{Two-fluid hydrostatic problem in a square domain}

The same square container of the previous example is considered assuming that the upper half is filled with a liquid of density $\rho=10^{-3} \mathrm{Kg} / \mathrm{m}^{3}$. The viscosity is the same for both fluids with $\mu=10^{-3} \mathrm{Ns} / \mathrm{m}^{-2}$. The body forces, the boundary conditions and the mesh are the same as for the previous example. The exact analytical solution is $\mathbf{v}=0$ in the whole container and a linear 


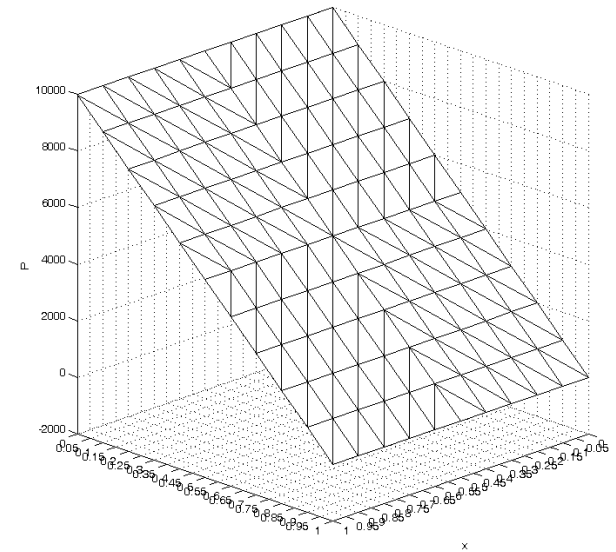

(a)

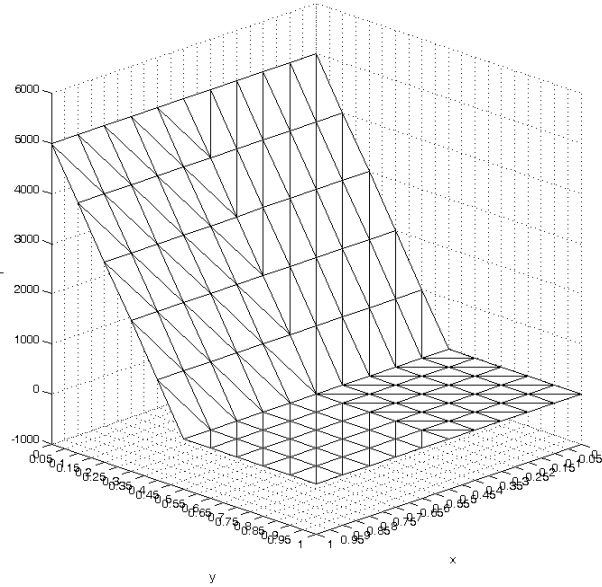

(b)

Figure 2. (a) Hydrostatic flow problem for a single fluid in square domain. (b) Two-fluid hydrostatic problem in square domain. Pressure distribution for both problems obtained with PLS (with and without BI), GLS (with and without BI) and OSS (using $\mathbf{T}$ and $\mathbf{T}_{d}$ ). Results for all methods coincide.

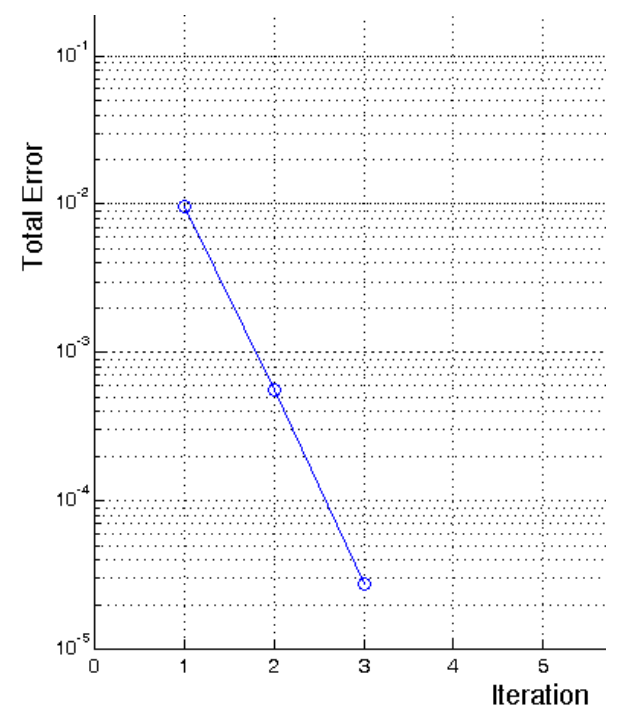

Figure 3. Two-fluid hydrostatic problem. Convergence of the PLS method (with and without BI).

distribution of the pressure ranging from $p=0$ at the top $\left(x_{2}=1.0 \mathrm{~m}\right)$ to $p=10^{-2} \mathrm{~Pa}$ at $x_{2}=0.5$ $\mathrm{m}$; and again a linear distribution of the pressure from $p=10^{-2} \mathrm{~Pa}$ at $x_{2}=0.5 \mathrm{~m}$ to $p=5000 \mathrm{~Pa}$ at $x_{2}=0$.

Results for the pressure distribution are shown in Figure 2b. Numerical results for all methods studied coincide.

The converged solution for the PLS method is obtained in three iterations. The convergence history is shown in Figure 3.

\subsection{Poiseuille flow in a trapezoidal domain}

A trapezoidal domain $\Omega$ is considered with corner nodes given by: $(0,0),(6,0),(4,2)$ and $(2,2)$. The domain is discretized with a mesh of $2 \times 10 \times 10$ 3-noded triangles (Figure 4). A parabolic profile for the horizontal velocity is prescribed at both the inlet and outlet lines. 


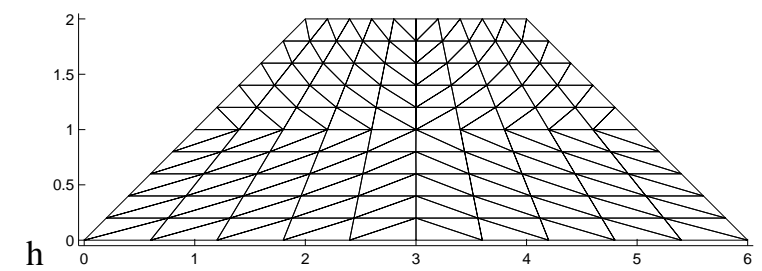

Figure 4. Trapezoidal domain. Symmetrical mesh of $2 \times 10 \times 103$-noded finite elements

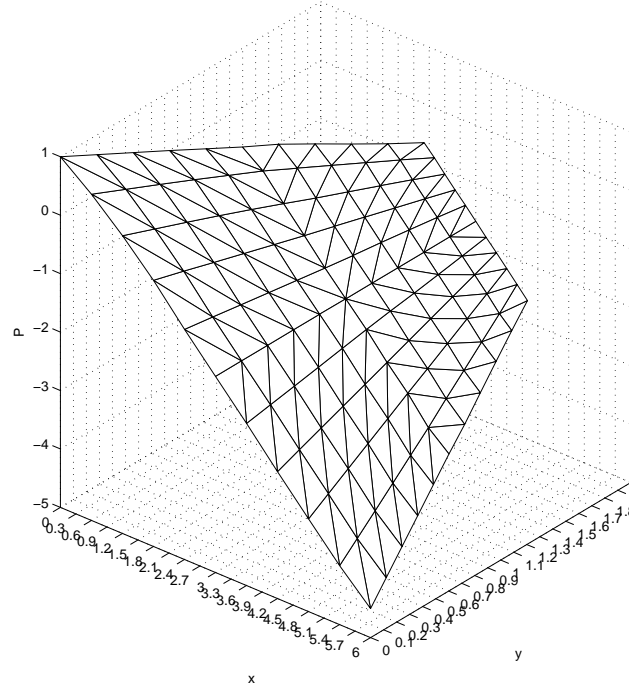

(a)

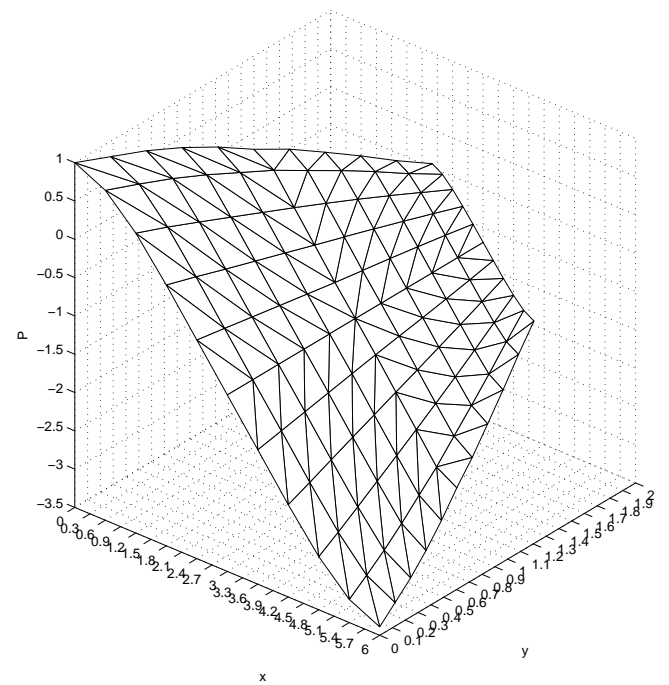

(b)

Figure 5. Poiseuille flow in a trapezoidal domain. (a) Pressure distribution obtained with PLS (with and without BI), GLS (with BI) and OSS (using $\mathbf{T}$ and $\mathbf{T}_{d}$ ). (b) Pressure distribution obtained with GLS without BI

Figure 5a shows the pressure distribution obtained with PLS method (with and without BI), the GLS method (with BI) and the OSS method (using $\mathbf{T}$ and $\mathbf{T}_{d}$ ). Results for all these methods coincide. Figure 5b shows the GLS results not including the BI term. Note the inaccuracies in the pressure distribution near the edge.

The same trend is observed for the results of the distribution of the $v_{1}$ velocity shown in Figure 6. Note the slight increase in accuracy obtained in the PLS method by including the BI term.

The convergence of the PLS solution with and without taking into account the BI terms is shown in Figure 7. The convergence improves when the BI terms are included ( 3 iterations versus 5 iterations).

\subsection{Lid a driven cavity problem}

The flow in a driven square cavity of $1 \times 1 \mathrm{~m}^{2}$ is studied.

The horizontal velocity on the top surface nodes has been prescribed to $v_{1}^{p}\left(x_{1}, 1\right)=1 \mathrm{~m} / \mathrm{s}$. The vertical velocity has also been prescribed to zero at all nodes on the top surface with the exception of the central node with coordinates $(0.5,1)$ which is left free to move in the vertical direction. The normal velocity at the bottom line and the two vertical walls has been prescribed to zero. The physical properties are $\rho=10^{-10} \mathrm{Kg} / \mathrm{m}^{3}, g=0 \mathrm{~N} / \mathrm{m}^{2}, \mu=1 \mathrm{Ns} / \mathrm{m}^{2}$.

It can be easily verified that, for the material properties chosen, the value of the stabilization parameter $\tau_{i}$ for the PLS method is approximately constant over the whole analysis domain and 


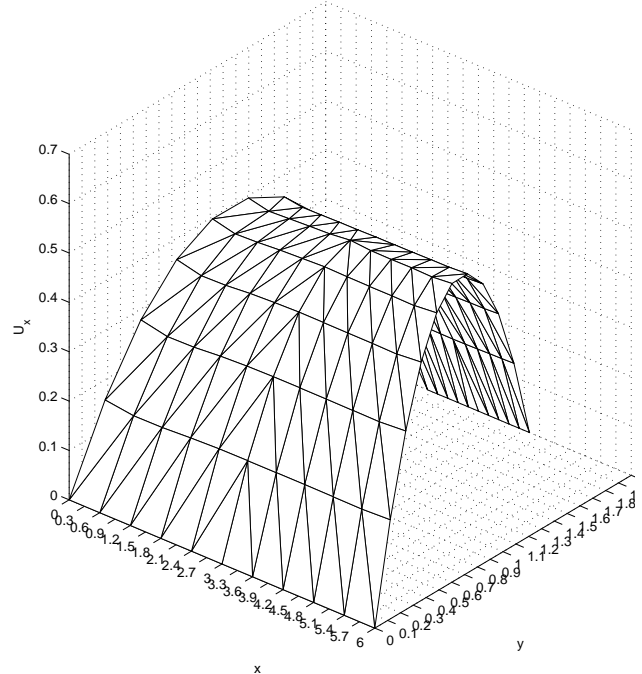

(a) PLS (without BI)

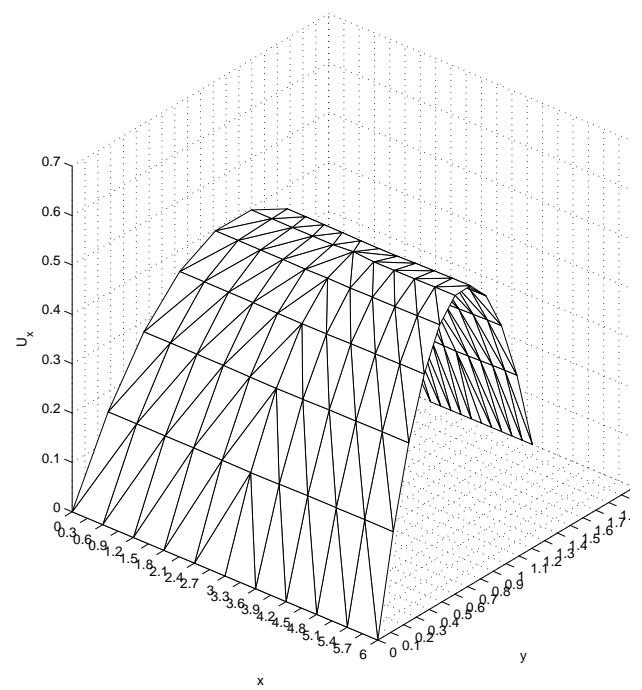

(c) PLS (with BI)

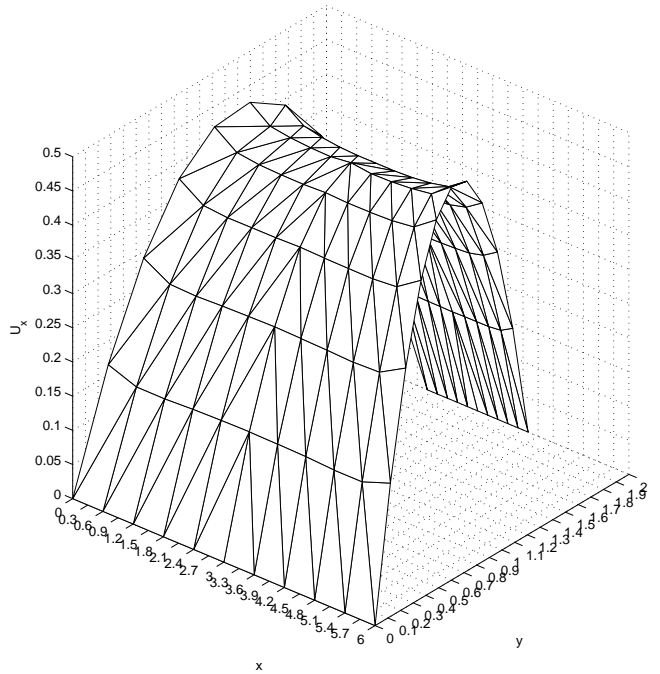

(b) GLS (without BI)

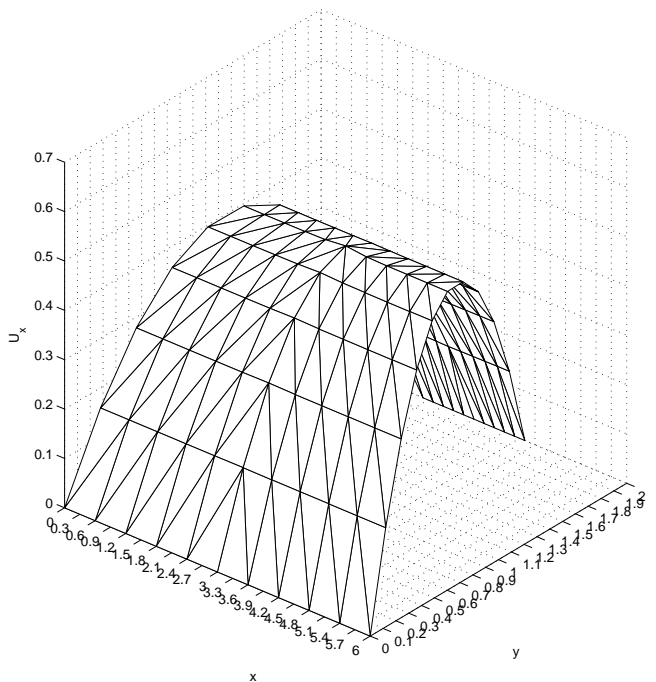

(d) GLS (with BI) and OSS (with $\mathbf{T}$ and $\mathbf{T}_{d}$ )

Figure 6. Poiseuille flow in a trapezoidal domain. Results for the velocity $v_{1}$ (denoted $u_{x}$ in the figure obtained with (a) PLS (without BI), (b) GLS (without BI), (c) PLS (with BI), (d) GLS (with BI) and OSS (with $\mathbf{T}$ and $\mathbf{T}_{d}$ )

equal to

$$
\tau_{i}=\tau \simeq \frac{h_{i}^{2}}{16 \mu}=\frac{10^{-2}}{16}=6.25 \times 10^{-3} \frac{m^{3} s}{K g}
$$

Figure 8 shows the pressure distributions in the cavity for all the methods studied using a $2 \times 20 \times 20$ mesh of 3-noded triangles. An analysis of the results of Figure 8 shows that (a) the PLS method captures better the singularity of the pressure values at the top corner nodes, (b) the effect of the BI terms is irrelevant for the PLS method but has a positive influence in the GLS method in terms of a better capture of the pressure singularity, (c) the diagonal form of matrix $\mathbf{T}$ introduce a slight diffusion in the OSS results. The pressure contour lines for the considered methods is shown in Figure 9. 

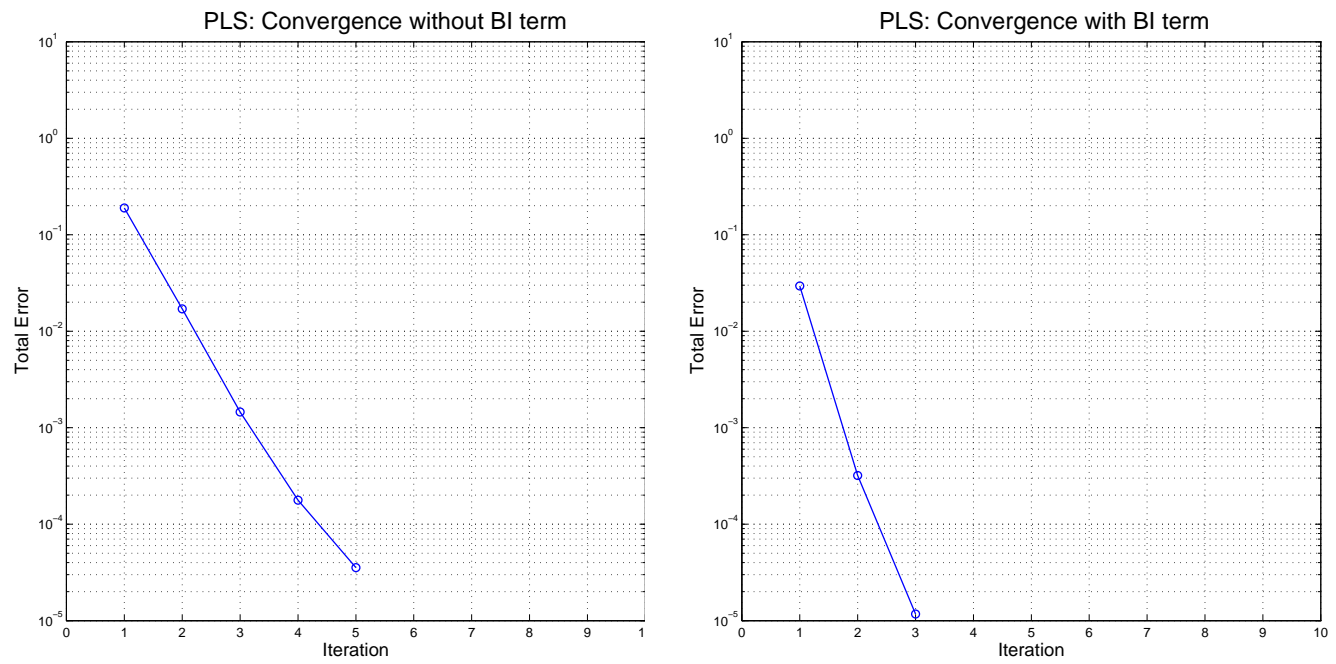

Figure 7. Poiseuille flow in a trapezoidal domain. Convergence of the PLS solution. (a) Without BI term. (b) With BI terms

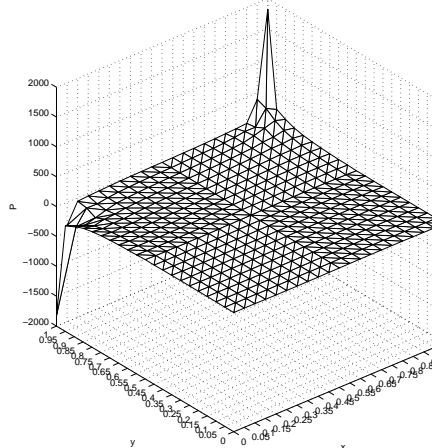

(a) PLS (without BI)

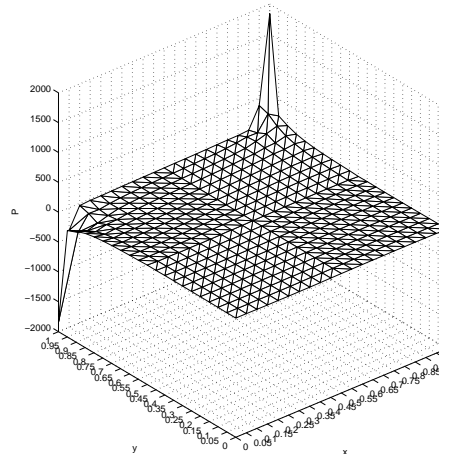

(d) PLS (with BI)

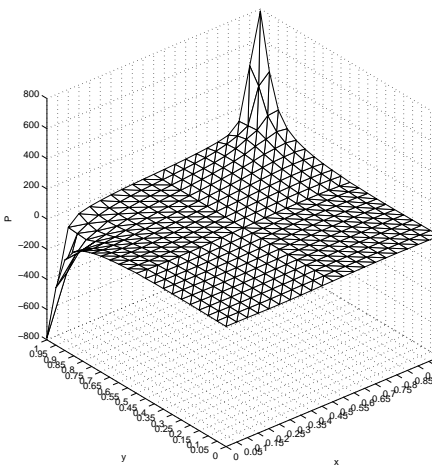

(b) GLS (without BI)

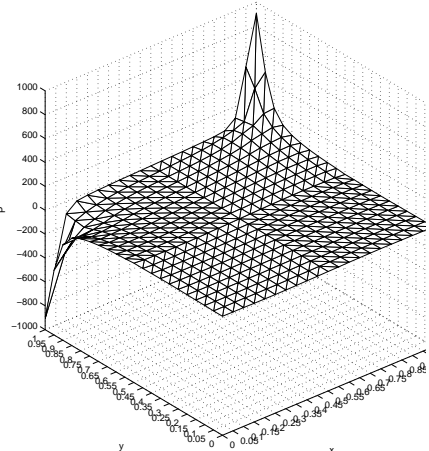

(e) GLS (with BI)

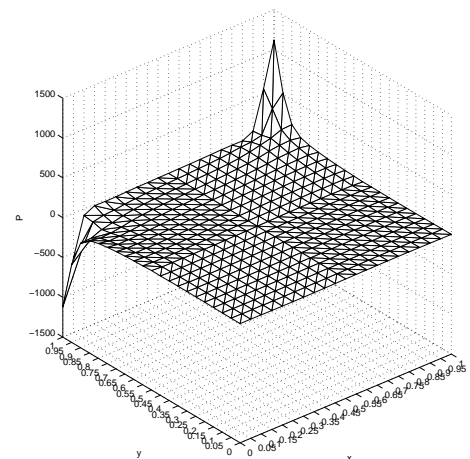

(c) OSS (with $\mathbf{T}_{d}$ )

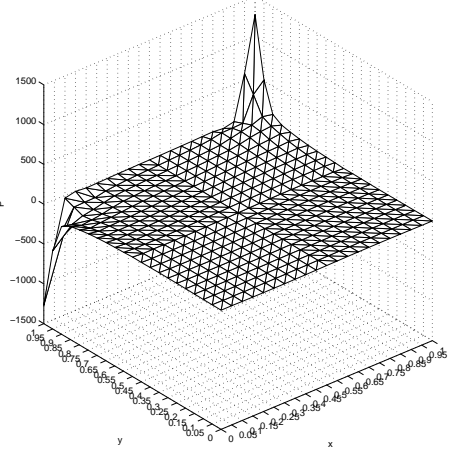

(f) OSS (with T)

Figure 8. Lid driven cavity problem. Pressure distribution results with (a) PLS (without BI). (b) GLS (without BI). (c) OSS (with $\mathbf{T}_{d}$ ). (d) PLS (with BI). (e) GLS (with BI). (f) OSS (with T )

Figure 10 shows the convergence of the PLS results. The convergence curve is practically the same accounting or not for the BI terms. Convergence is slower in this case due to the pressure singularity at the top corners. 


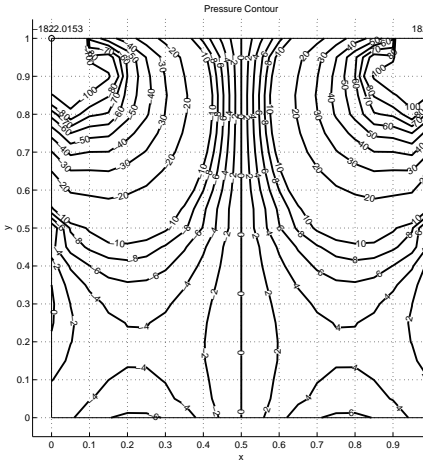

(a) PLS (without BI)

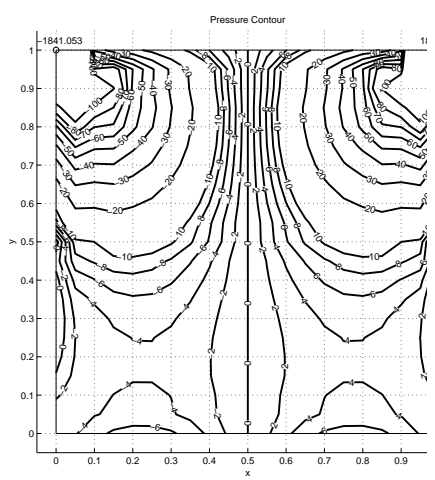

(d) PLS (with BI)

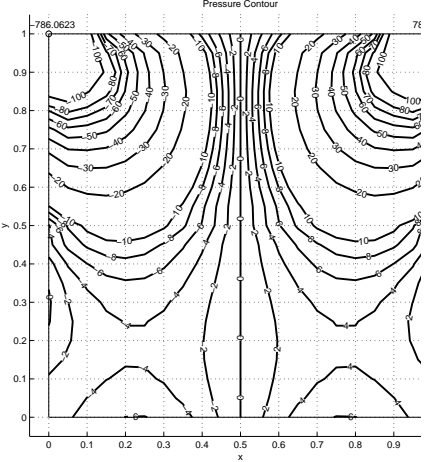

(b) GLS (without BI)

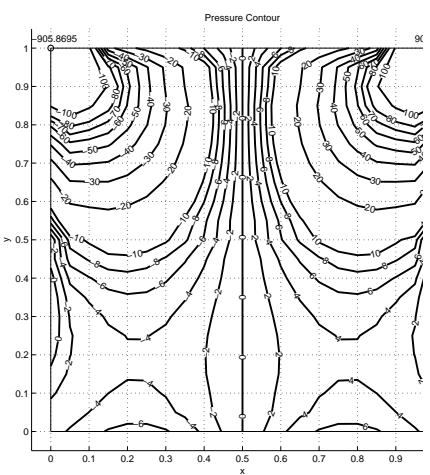

(e) GLS (with BI)

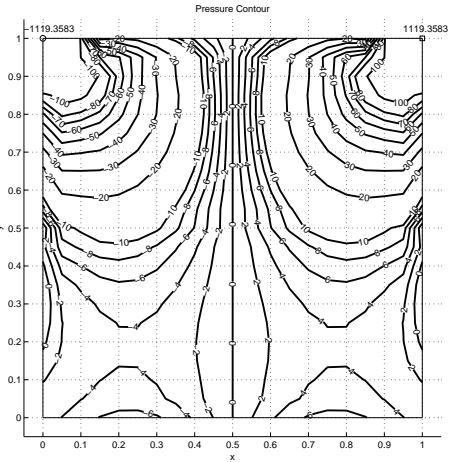

(c) OSS (with $\mathbf{T}_{d}$ )

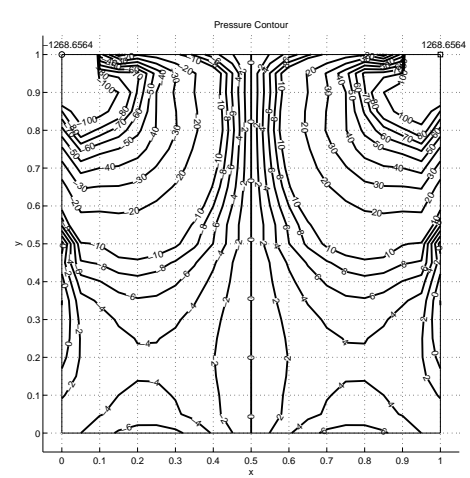

(f) OSS (with T)

Figure 9. Lid driven cavity problem. Pressure contours with (a) PLS (without BI). (b) GLS (without BI). (c) OSS (with $\mathbf{T}_{d}$ ). (d) PLS (with BI). (e) GLS (with BI). (f) OSS (with T)

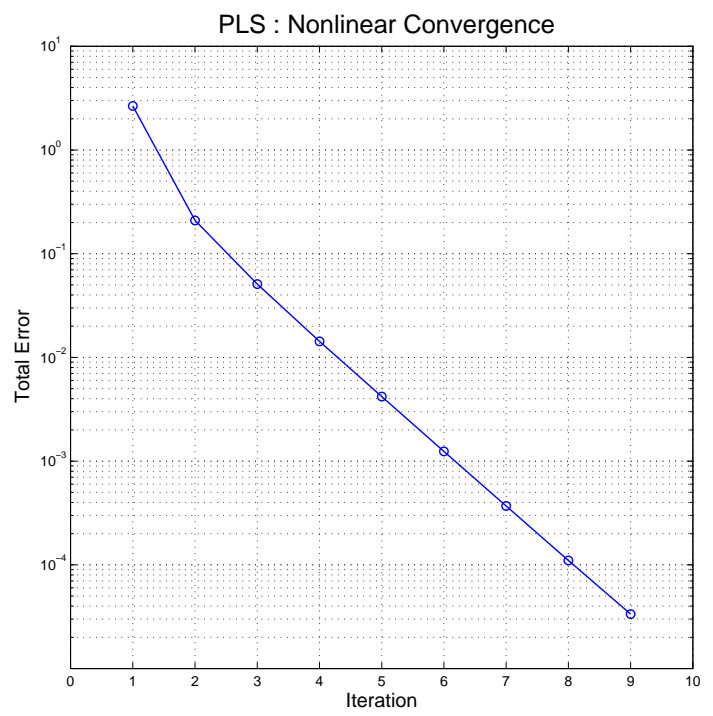

Figure 10. Lid driven cavity problem. Convergence of PLS results. Convergence curve with or without BI terms are similar

\subsection{Manufactured flow problem in a trapezoidal domain}

The trapezoidal domain of Figure 4 is discretized by a series of symmetrical mesh consisting of $2 \times n \times n$ 3-noded triangular elements and using $n \in\{10,12,14,16,18,20,24,32,36,40\}$. The 
following relative error norms are used to study the convergence rates of the considered methods.

$$
\begin{gathered}
e_{v H^{1}}^{h}=\frac{\|\mathbf{v}-\overline{\mathbf{v}}\|_{1}}{\|\mathbf{v}\|_{1}}=\frac{\sqrt{\int_{\Omega} \boldsymbol{\nabla}(\mathbf{v}-\overline{\mathbf{v}}): \nabla(\mathbf{v}-\overline{\mathbf{v}}) \mathrm{d} \Omega}}{\sqrt{\int_{\Omega} \boldsymbol{\nabla} \mathbf{u}: \nabla \mathbf{u} \mathrm{d} \Omega}} \\
e_{p L^{2}}^{h}=\frac{\|p-\bar{p}\|_{0}}{\|p\|_{0}}=\frac{\sqrt{\int_{\Omega}(p-\bar{p})^{2} \mathrm{~d} \Omega}}{\sqrt{\int_{\Omega} p^{2} \mathrm{~d} \Omega}}
\end{gathered}
$$

where, $(\overline{\mathbf{v}}, \bar{p})$ is the finite element approximation of the exact solution $(\mathbf{v}, p)$. The numerical solutions corresponding to the GLS, PLS, PLS $+\pi$ and OSS methods, are compared with the following solutions: the nodally exact interpolant denoted by $\left(I_{h} \mathbf{v}, I_{h} p\right)$ and the best approximation (BA) with respect to the $L^{2}$ norm (for pressure) and the $H^{1}$ semi-norm (for velocity) denoted by $P_{h}^{0} p$ and $P_{h}^{1} \mathbf{v}$ respectively. The solutions $I_{h} \mathbf{v}, I_{h} p, P_{h}^{0} p$ and $P_{h}^{1} \mathbf{v}$ can be found as shown in Eq.(83).

$$
\begin{gathered}
I_{h} \mathbf{v}:=N^{a} \mathbf{v}^{a} ; \quad I_{h} p:=N^{a} p^{a} \\
\int_{\Omega} q^{h}\left(p-P_{h}^{0} p\right) \mathrm{d} \Omega=0 \forall q^{h} \in Q^{h} \Rightarrow\left\|p-P_{h}^{0} p\right\|_{0} \leq\|p-\bar{p}\|_{0} \forall \bar{p} \in Q^{h} \\
\int_{\Omega} \nabla \mathbf{v}^{h}: \nabla\left(\mathbf{v}-P_{h}^{1} \mathbf{v}\right) \mathrm{d} \Omega=0 \forall \mathbf{v}^{h} \in V^{h} \Rightarrow\left\|\mathbf{u}-P_{h}^{1} \mathbf{v}\right\|_{1} \leq\|\mathbf{v}-\overline{\mathbf{v}}\|_{1} \forall \overline{\mathbf{v}} \in V^{h}
\end{gathered}
$$

where, $\left(\mathbf{v}^{a}, p^{a}\right)$ represent the nodal values of the exact solution $(\mathbf{v}, p), Q^{h} \subset L^{2}(\Omega)$ and $V^{h} \subset$ $H_{0}^{1}(\Omega)$. In the current example $Q^{h}$ and $V^{h}$ are the solution spaces spanned by the 3-noded triangle shape functions.

Consider a manufactured flow problem in which choosing the force term $\mathbf{f}=\left(f_{1}, f_{2}\right)$, with $f_{1}=\mu(6 x-17), f_{2}=0$, we have the exact solution to the Stokes problem as $\mathbf{v}=\left(v_{1}, v_{2}\right)$, with $v_{1}=y(2-y) / 2, v_{2}=0$ and $p=\mu\left(3 x^{2}-18 x+1\right)$. Note that changing the magnitude of $\mu$, any inf-sup stable Galerkin-FEM will give numerical solutions that would scale proportional to the exact solution. Thus, for this manufactured problem, the relative errors $e_{v H^{1}}^{h}$ and $e_{p L^{2}}^{h}$ will be independent of $\mu$.

The $e_{v H^{1}}^{h}$ and $e_{p L^{2}}^{h}$ convergence rates for the GLS, PLS, PLS $+\pi$ and OSS methods using $\mu=1$ are shown in Figure 11.

Figure 11(a) illustrates the $e_{v H^{1}}^{h}$ error lines for the considered methods. The error line of the GLS method is slightly shifted above the error lines of $I_{h} \mathbf{v}$ (label: 'Interpolant') and $P_{h}^{1} \mathbf{v}$ (label: ' $H^{1}$ BA'). The error line of the OSS method shows a slight deviation from those of $I_{h} \mathbf{v}$ and $P_{h}^{1} \mathbf{v}$, but it quickly merges with the later error lines on mesh refinement. The error line of the PLS method shows an improvement over the GLS method on coarse meshes but this advantage is lost on finer meshes wherein the two error lines merge. The error line of the PLS $+\pi$ method practically coincides with the error lines of $I_{h} \mathbf{v}$ and $P_{h}^{1} \mathbf{v}$.

Figure 11(b) illustrates the $e_{p L^{2}}^{h}$ error lines for the considered methods. The error line of the GLS method not only shows the greatest deviation from the error lines of $I_{h} p$ (label: 'Interpolant') and $P_{h}^{0} p$ (label: ' $L^{2}$-BA') but also a sub-optimal convergence rate. The OSS method on the other hand show a convergence rate similar to that of $I_{h} p$ and $P_{h}^{0} p$ and the improvement over the GLS method is clear. The PLS and PLS $+\pi$ methods show convergence rates similar to that of the GLS and the OSS methods respectively. Nevertheless, the error lines of the former two methods are located closer to the error lines of $I_{h} p$ and $P_{h}^{0} p$ showing improved accuracy in the considered norms. The increase in accuracy is particularly relevant for the PLS $+\pi$ method as clearly seen in Figure $11 \mathrm{~b}$.

The PLS results for each of the meshes studied were obtained in 7-9 iterations whereas only 4 iterations were needed to obtain each of the PLS $+\pi$ solutions. 


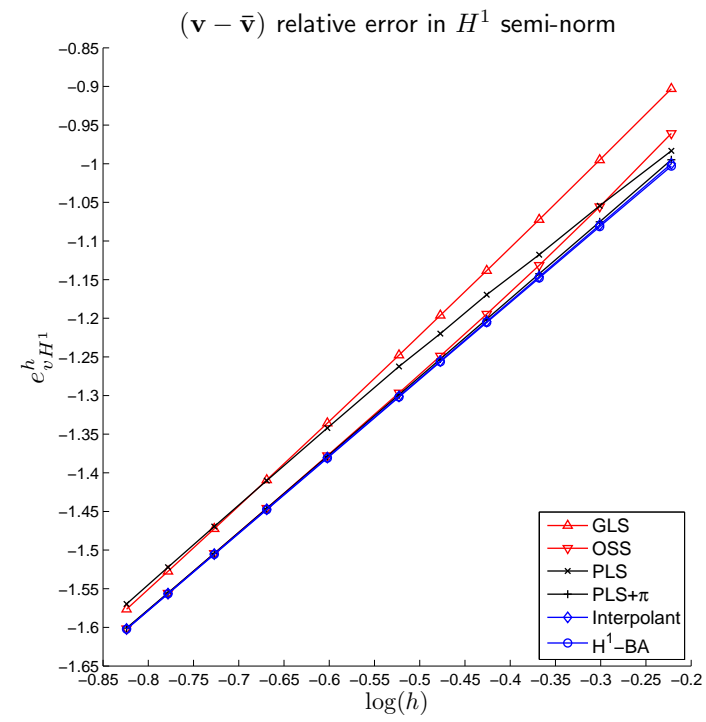

(a)

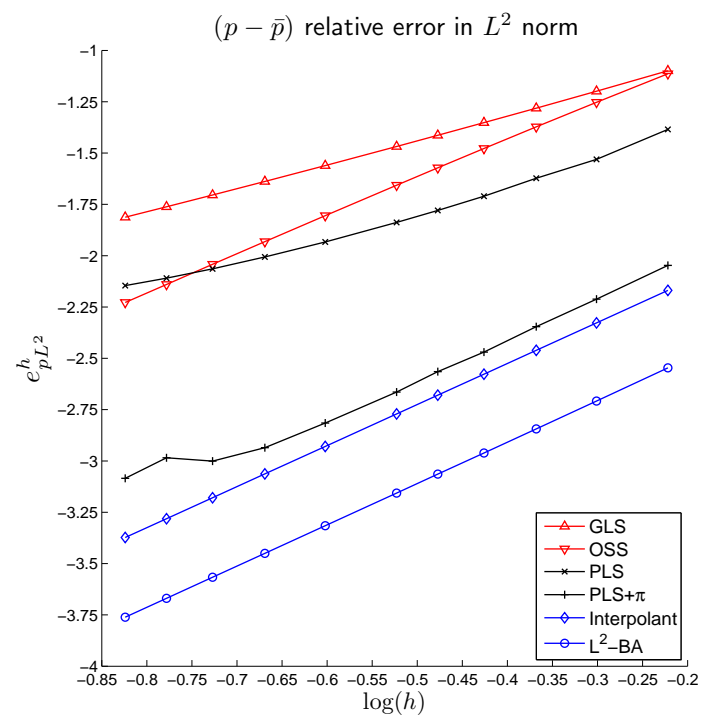

(b)

Figure 11. Convergence rates for the GLS, PGP, PLS and PLS $+\pi$ methods: (a) the relative errors in the velocity $\left(e_{v H^{1}}^{h}\right)$ and (b) the relative errors in the pressure $\left(e_{p L^{2}}^{h}\right)$

\section{CONCLUSIONS}

We have presented a family of stabilized finite element (FE) methods derived via first and second order the finite calculus (FIC) procedures. We have shown that several well known existing stabilized FE methods such as the penalty technique, the Galerkin Least Square (GLS) method, the Pressure Gradient Projection (PGP) method and the Orthogonal Sub-Scales (OSS) method are recovered from the general residual-based FIC stabilized form. New stabilized FE methods such as the Pressure Laplacian Stabilization (PLS) and the PLS $+\pi$ method with consistent non linear forms of the stabilization parameters have been derived. The distinct feature of the PLS and PLS+ $\pi$ methods is that the stabilization terms depend on the discrete residuals of the momentum and the incompressibility equations.

The numerical results obtained for the Stokes problems solved in this work show that the PLS method and, in particular, the PLS $+\pi$ method provide accurate solutions that improve in several cases the results of the traditional GLS and OSS methods. Results presented in [46] indicate that the PLS methods has also a superior performance than the PGP method for some problems.

The prize to be paid for this increase in accuracy is the higher computational cost associated to the non linear solution which is intrinsic to the PLS method. The potential and advantages of the PLS method will be clearer for transient problems solved via staggered schemes or for non linear flow problems which invariably require an iterative scheme.

\section{ACKNOWLEDGEMENTS}

Support for this research was provided by the SEDUREC project of the Consolider Programme of the Ministry of Science and Innovation of Spain and from the REALTIME and SAFECON projects of the European Research Council of the European Commission.

The second author acknowledges the economic support received through the FI pre-doctoral grant from the Department of Universities, Research and Information Society (Generalitat de Catalunya) and the European Social Fund. The work of the last author was partly supported through a Visiting Professor fellowship awarded by the Spanish Ministerio of Educación y Cultura. 


\section{REFERENCES}

1. Donea J, Huerta A. Finite Element Method for Flow Problems. J. Wiley, 2003.

2. Zienkiewicz OC, Taylor RL, Nietharasu P. The Finite Element Method. Fluid Mechanics, Volume 3, Elsevier, 2005.

3. Brooks AN, Hughes TJR. Streamline upwind Petrov-Galerkin formulation for convective dominated flows with particular emphasis on the incompressible Navier-Stokes equations. Computer Methods in Applied Mechanics and Engineering 1982; 32:199-259.

4. Hughes TJR, Franca LP, Balestra M. A new finite element formulation for computational fluid dynamics. V Circumventing the Babuska-Brezzi condition: A stable Petrov-Galerkin formulation of the Stokes problem accomodating equal order interpolations. Computer Methods in Applied Mechanics and Engineering 1986; 59:8599.

5. Franca LP, Frey SL. Stabilized finite element methods: II. The incompressible Navier-Stokes equations. Computer Methods in Applied Mechanics and Engineering 1992; 99:209-233.

6. Hughes TJR, Hauke G, Jansen K. Stabilized finite element methods in fluids: Inspirations, origins, status and recent developments. In Recent Developments in Finite Element Analysis. A Book Dedicated to Taylor RL. Hughes TJR, Oñate E, Zienkiewicz OC (eds), International Center for Numerical Methods in Engineering (CIMNE), Barcelona, Spain, 1994; 272-292.

7. Tezduyar TE, Mittal S, Ray SE, Shih R. Incompressible flow computations with stabilized bilinear and linear equal order interpolation velocity-pressure elements. Computer Methods in Applied Mechanics and Engineering 1992; 95:221-242.

8. Droux J-J, Hughes TJR. A boundary integral modification of the Galerkin least squares formulation for the Stokes problem. Computer Methods in Applied Mechanics and Engineering 1994; 113:173-182.

9. Storti M, Nigro N, Idelsohn SR. Steady state incompressible flows using explicit schemes with an optimal local preconditioning. Computer Methods in Applied Mechanics and Engineering 1995; 124:231-252.

10. Cruchaga MA, Oñate E. A finite element formulation for incompressible flow problems using a generalized streamline operator. Computer Methods in Applied Mechanics and Engineering 1997; 143:49-67.

11. Codina R, Vazquez M, Zienkiewicz OC. A general algorithm for compressible and incompressible flow - Part III. The semi-implicit form. International Journal for Numerical Methods in Fluids 1998; 27:13-32.

12. Cruchaga MA, Oñate E. A generalized streamline finite element approach for the analysis of incompressible flow problems including moving surfaces. Computer Methods in Applied Mechanics and Engineering 1999; 173:241255.

13. Tezduyar TE, Osawa Y. Finite element stabilization parameters computed from element matrices and vectors. Computer Methods in Applied Mechanics and Engineering 2000; 190:411-430.

14. Hughes TJR, Mazzei L, Jansen KE. Large eddy simulation and the variational multiscale method. Computing and Visualization in Science 2000; 3:47-59.

15. Codina R, Blasco J. A finite element formulation for the Stokes problem allowing equal velocity-pressure interpolation. Computer Methods in Applied Mechanics and Engineering 1997; 143:373-391.

16. Codina R, Blasco J. Stabilized finite element method for the transient Navier-Stokes equations based on a pressure gradient operator. Computer Methods in Applied Mechanics and Engineering 2000; 182:277-301.

17. Codina R. Stabilization of incompressibility and convection through orthogonal sub-scales in finite element methods. Computer Methods in Applied Mechanics and Engineering 2000; 190:1579-1599.

18. Codina R, Zienkiewicz OC. CBS versus GLS stabilization of the incompressible Navier-Stokes equations and the role of the time step as stabilization parameter. Communications Numerical Methods in Engineering 2002; 18(2):99-112.

19. Dohrmann CR, Bochev PB, A stabilized finite element method for the Stokes problem based on polynomial pressure projections. Int. J. Numer. Meth. Fluids 2004; 46:183201.

20. Codina R, Principe J, Guasch O, Badia S. Time dependent subscales in the stabilized finite element approximation of incompressiblity flow problems. Communications Numerical Methods in Engineering 2007; 196(2):2413-2430.

21. Badia S, Codina R. On a multiscale approach to the transient Stokes problem. Transient subscales and anistropic space-time discretization. Applied Mathematics and Computation 2009; 207:415-433.

22. Oñate E. Derivation of stabilized equations for advective-diffusive transport and fluid flow problems. Computer Methods in Applied Mechanics and Engineering 1998; 151:233-267.

23. Oñate E. A stabilized finite element method for incompressible viscous flows using a finite increment calculus formulation. Computer Methods in Applied Mechanics and Engineering 2000; 182(1-2):355-370.

24. Tezduyar TE. Computation of moving boundaries and interfaces and stabilization parameters. International Journal for Numerical Methods in Fluids, 2003; 43:555-575.

25. Akin JE, Tezduyar TE, Ungor M, Mittal S. Stabilization parameters and smagorinsky turbulence model. Journal of Applied Mechanics 2003; 70:2-9.

26. Akin JE, Tezduyar TE. Calculation of the advective limit of the SUPG stabilization parameter for linear and higherorder elements. Computer Methods in Applied Mechanics and Engineering 2004; 193:1909-1922.

27. Bazilevs Y, Calo Tezduyar TE, Hughes, TJR. YZ? Discontinuity-capturing for advection-dominated processes with application to arterial drug delivery. International Journal for Numerical Methods in Fluids 2007; 54:593-608.

28. Hsu M-C, Bazilevs Y, Calo VM, Tezduyar TE, Hughes, TJR. Improving stability of stabilized and multiscale formulations in flow simulations at small time steps. Computer Methods in Applied Mechanics and Engineering 2010; 199:828-840.

29. Oñate E, García J. A finite element method for fluid-structure interaction with surface waves using a finite calculus formulation. Computer Methods in Applied Mechanics and Engineering 2001; 191:635-660.

30. Oñate E, Taylor RL, Zienkiewicz OC, Rojek J. A residual correction method based on finite calculus. Engineering Computations 2003; 20(5/6):629-658.

31. Oñate E. Possibilities of finite calculus in computational mechanics. International Journal for Numerical Methods in Engineering 2004; 60(1):255-281. 
32. Oñate E, Idelsohn SR, Del Pin F, Aubry R. The particle finite element method. An overview. International Journal of Computational Methods 2004; 1(2):267-307.

33. Idelsohn SR, Oñate E, Del Pin F. The Particle Finite Element Method: a powerful tool to solve incompressible flows with free-surfaces and breaking waves. International Journal for Numerical Methods in Engineering 2004; 61:964-989.

34. Oñate E, Rojek J, Taylor RL Zienkiewicz OC. Finite calculus formulation for incompressible solids using linear triangles and tetrahedra. International Journal for Numerical Methods in Engineering 2004; 59:1473-1500.

35. Oñate E, Valls A, García J. FIC/FEM formulation with matrix stabilizing terms for incompressible flows at low and high Reynolds numbers. Computational Mechanics 2006; 38(4-5):440-455.

36. Oñate E, García J, Idelsohn SR, Del Pin F. FIC formulations for finite element analysis of incompressible flows. Eulerian, ALE and Lagrangian approaches. Computer Methods in Applied Mechanics and Engineering 2006; 195(23-24):3001-3037.

37. Oñate E, Valls A, García J. Modeling incompressible flows at low and high Reynolds numbers via a finite calculusfinite element approach. Journal of Computational Physics 2007; 224:332-351.

38. Oñate E, Idelsohn SR, Celigueta MA, Rossi R Advances in the particle finite element method for the analysis of fluid-multibody interaction and bed erosion in free surface flows. Comput. Methods Appl. Mech. Engrg. 2008, 197(19-20): 1777-1800.

39. Idelsohn SR, Mier-Torrecilla M, Oñate E. Multi-fluid flows with the Particle Finite Element Method. Comput Methods Appl Mech Engrg. 2009; 198:2750-2767.

40. Idelsohn SR, Del Pin F, Rossi R, Oñate E. Fluid-structure interaction problems with strong added-mass effect. International Journal for Numerical Methods in Engineering 2009; 80(10):1261-1294.

41. Zienkiewicz OC, Taylor RL, The Finite Element Method for Solid and Structural Mechanics, Volume 2, Butterworth-Heinemann, 2005.

42. Idelsohn SR, Marti J, Limache A, Oñate E. Unified Lagrangian formulation for elastic solids and incompressible fluids: Application to fluid-structure interaction problems via the PFEM. Comput Methods Appl Mech Engrg. 2008; 197:1762-1776.

43. Idelsohn SR, Oñate E. The challenge of mass conservation in the solution of free-surface flows with the fractional-step method: Problems and solutions. Communications in Numerical Methods in Engineering 2008; DOI: $10.1002 / \mathrm{cnm} .1216$

44. Idelsohn SR, Mier-Torrecilla M, Nigro N, Onate E. On the analysis of heterogeneous fluids with jumps in the viscosity using a discontinuous pressure field. Comput. Mech. 2010; 46 (1):115-124.

45. de Mier Torrecilla M. Numerical Simulation of Multi-Fluid Flows with the Particle Finite Element Method. Ph.D. Thesis, Technical University of Catalonia (UPC), July 2010.

46. Oñate E, Idelsohn SR, Felippa C. Consistent pressure Laplacian stabilization for incompressible continua. International Journal for Numerical Methods in Engineering. Submitted May 2010.

47. Nadukandi P, Stabilized finite element methods for convection-diffusion-reaction and fluid flow problems. Ph.D. Thesis, Technical University of Catalonia (UPC), 2011. 